\title{
Insights into the dynamics of supercritical water flow using a two-phase approach
}

Cite as: Phys. Fluids 33, 043304 (2021); https://doi.org/10.1063/5.0042935

Submitted: 05 January 2021. Accepted: 13 March 2021 . Published Online: 07 April 2021

Piyush Mani Tripathi, and (D) Saptarshi Basu
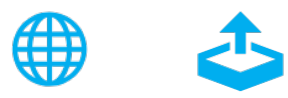

Export Citation

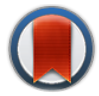

View Online

CrossMark

\section{ARTICLES YOU MAY BE INTERESTED IN}

Universal spatio-topological control of crystallization in sessile droplets using non-intrusive vapor mediation

Physics of Fluids 33, 012101 (2021); https://doi.org/10.1063/5.0037120

Insights on drying and precipitation dynamics of respiratory droplets from the perspective of COVID-19

Physics of Fluids 32, 123317 (2020); https://doi.org/10.1063/5.0037360

Equilibrium approach for modeling erosional failure of granular dams

Physics of Fluids 33, 043306 (2021); https://doi.org/10.1063/5.0039140 


\title{
Insights into the dynamics of supercritical water flow using a two-phase approach
}

\author{
Cite as: Phys. Fluids 33, 043304 (2021); doi: 10.1063/5.0042935 \\ Submitted: 5 January 2021 - Accepted: 13 March 2021 . \\ Published Online: 7 April 2021
}

Piyush Mani Tripathi and Saptarshi Basu ${ }^{a)}$ (iD

\author{
AFFILIATIONS \\ Department of Mechanical Engineering, Indian Institute of Science, Bangalore 560012 India \\ a) Author to whom correspondence should be addressed: sbasu@iisc.ac.in
}

\begin{abstract}
A two-phase approach has been proposed to study the supercritical flow with heat transfer deterioration (HTD) phenomena so that an analogy can be derived between subcritical flow boiling and supercritical heat transfer. The volume of fluid multiphase model has been used to analyze the flow, and the simulation result reasonably predicts the wall temperature peaks. Moreover, the velocity and turbulent kinetic energy profiles at different axial locations explain the occurrence of HTD. The parametric study of the thermophysical properties revealed that the density variation is the primary cause of HTD in supercritical flows. Leaning onto this observation, the current study focuses on the forces generated due to the density variation. It suggests that for no HTD, buoyancy and inertia forces have to be of comparable magnitude throughout the flow. Mapping of volume fraction variable reveals a sudden jump in the lighter phase thickness near the wall at the site of HTD, which is also reflected as a maximum in the plot of nondimensional two-phase interface distance from the wall (P). However, this observation is only restricted to HTD caused by buoyancy. This can be used to draw analogy with the phenomenon of film boiling in subcritical fluids. In the end, a theoretical expression has been conceptualized for computing the phase boundary distance from the wall (h), which can serve as a fundamental length scale in supercritical flows as it marks the region of highest property gradient near the wall.
\end{abstract}

Published under license by AIP Publishing. https://doi.org/10.1063/5.0042935

\section{INTRODUCTION}

The phase transition above the critical point and its dissipation has captivated researchers all around the world for a very long time. This curiosity is mainly guided by the power plant sector, where supercritical fluids (SF) (fluid at a pressure higher than the critical value) like water can be used to increase efficiency significantly. However, inadequate knowledge and lack of predictability of SF flow and heat transfer behavior pose the most prominent challenge in designing a fail-safe supercritical power plant. Besides, the use of hydrocarbons like methane as an alternative fuel in liquid rocket propulsion has contributed to the recent surge in SF flow research.

Several studies have been conducted at the supercritical pressure (higher than critical value), which has shown a drastic variation in the thermo-physical properties of the fluid across a very narrow region. This region is generally known as the pseudocritical line or "widom line" emanating from the critical point into the supercritical domain. The widom line can be defined in various ways, such as the locus of a state with maximum response function (like specific heat) or the line joining the inflection point of the density variation for a given pressure. Interestingly, this pseudocritical line demarcates the fluid between liquid-like and vapor-like behavior. For example, supercritical water
(SCW) is distinguished ${ }^{1}$ into liquid-like supercritical water (LSCW) and vapor-like supercritical water (VSCW) as shown in Fig. 1.

Density may seem homogenous from the macroscopic point of view but there exists nonhomogeneity from a microscopic point of view. However, with an increase in pressure and temperature in the supercritical range, ${ }^{2}$ size of liquid like bundles in vapor like field decreases. This results in a change in the behavior of the whole system mimicking a vapor field with high degree of interaction. Although this understanding existed long ago, supercritical fluid has always been perceived as a single phase with property variation to understand the flow dynamics.

Contrary to this, the recent works ${ }^{3,4}$ on the transition of states across the pseudocritical line have challenged the traditional belief that supercritical fluid consists of a single phase. Banauti ${ }^{3}$ has demonstrated the prevalence of pseudo boiling phenomena upon crossing the widom line, which is analogous to subcritical phase change. Later on, $\mathrm{he}^{4}$ suggested a modified phase diagram by delineating the boundary based on the physical attributes of the fluid in the supercritical region.

Surprisingly, the analogy between subcritical phase-change and SF flow dates back to the second half of the 20th century. Initially, a few experimental studies ${ }^{5-8}$ focused on heat transfer characteristics of 

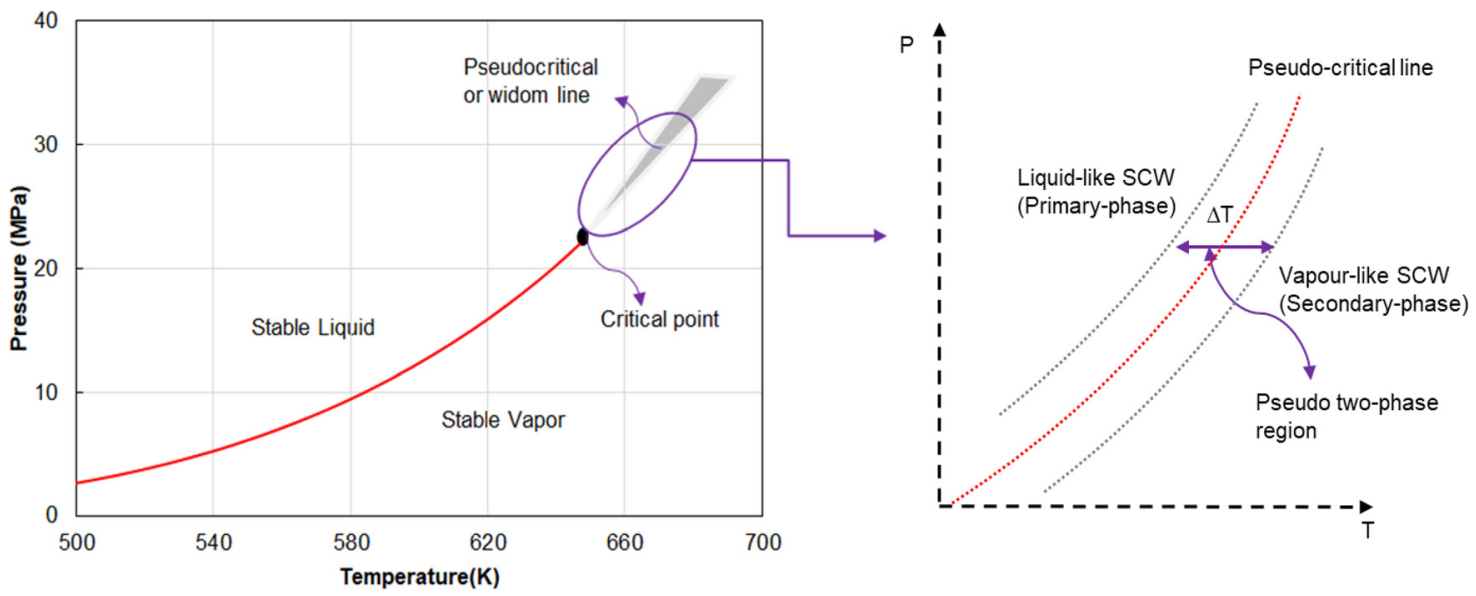

FIG. 1. Pressure vs temperature ( $\mathrm{p}-\mathrm{T})$ for water, including a schematic of the pseudo-two-phase region. The shaded region in the $\mathrm{p}-\mathrm{T}$ curve marks the pseudocritical region. The right side of the figure is the zoomed-in image (not drawn to scale) of the widom region, explaining the phase and their boundary in the proposed pseudophase change process.

supercritical fluid unanimously reported heat transfer deterioration (HTD) or heat transfer enhancement (HTE). Shitsman ${ }^{5}$ was the first to mention that the HTD occurred due to a phenomenon similar to film-boiling. Meanwhile, Ackerman ${ }^{7}$ coined the word "pseudoboiling" to underline the analogy of heat transfer variation of SF flow to normal boiling processes. However, an alternate explanation based on the buoyancy forces originating out of the variation of thermo-physical properties across the widom line came to light later on. Figure 2 depicts the properties of Supercritical water (SCW) as a function of temperature at a pressure of $25.3 \mathrm{MPa}$.

Shitsman ${ }^{9}$ argued that the source of HTD might be due to a decline in transverse turbulent velocity fluctuation which propagates due to radial density change. Hall and Jackson ${ }^{10-12}$ asserted that the

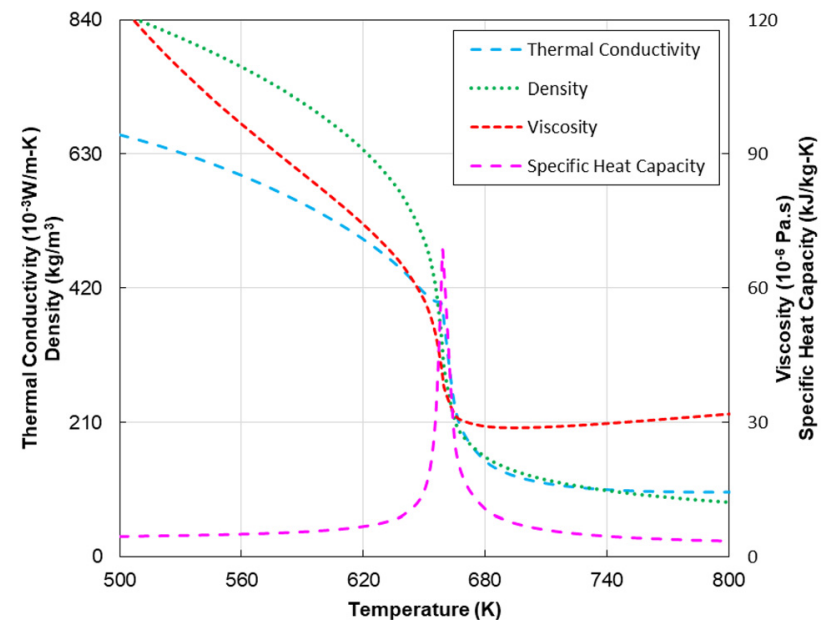

FIG. 2. Properties of Supercritical water at $25.3 \mathrm{MPa}$ (NIST miniREFPROP). The range of temperature in which the major chunk of variation occurs is same and very narrow for all the properties. Besides, the maximum of the specific heat can be perceived as a reflection of phase change happening at the microscopic level. buoyancy force manipulates the velocity of the fluid to reduce the turbulence near the wall. Nonetheless, few pieces of evidence contradict these explanations as HTD even occurs in the absence of buoyancy forces at certain flow conditions. For instance, Shiralkar and Griffith ${ }^{13}$ reported decline in heat transfer for upward as well as downward flow. This paradox was resolved later when the origin of such HTD was ascribed to the acceleration effect instead of buoyancy. Numerical studies ${ }^{14-20}$ have been performed for different SF flow conditions corresponding to preexisting experimental setups and have shown consistency in terms of results by predicting HTD and HTE. Similar to the experimental analysis, these studies have also found either buoyancy or acceleration effects to be the main reason for HTD or HTE occurrence.

The first explanation of the HTD ${ }^{5,7}$ was theorized based on the phase-change process where maxima are the result of the phenomena similar to boiling in subcritical flows. However, the two-phase aspect of supercritical fluid flow remains untouched. The recent review studies $^{21,22}$ on HTD phenomena in SF flow have summarized all the experimental and numerical work, where it can be seen that SF flow research is still restricted to the idea of treating it as a single phase with varying properties, for example, in the case of SCW, as shown in Fig. 2. No attempt has been made to verify the speculation that HTD in the SF (near the critical pressure) flow has a resemblance to boiling phenomena. In other words, there is no research work reported in the open literature that can demonstrate the analogy of subcritical flow boiling to supercritical heat transfer. This lacuna is the primary motivation of the presented work. So, a multiphase numerical model has been implemented, where the flow is treated as a two-phase flow undergoing phase change across the pseudocritical line, as shown in Fig. 1.

It is to be noted that if one can show the existence of pseudoflow boiling, then the concepts and understanding of the extensively explored (Analytically, experimentally, and numerically) boiling physics can be directly employed for SF flow. This implication can be clearly illustrated by the recent work, ${ }^{23,24}$ where criteria for the onset of HTD and heat transfer correlations are developed for supercritical 
$\mathrm{CO}_{2}$ and water, respectively. Interestingly, the common ground shared by both these studies is the assumption of pseudoboiling in SF heat transfer. So, the proposed research will boost the credibility of such studies and will give further motivation to pursue similar work. For example, the recent work on the subcritical film boiling ${ }^{25-27}$ can be relevant to SF flow because these studies have investigated the combined effect of buoyancy and inertia on heat transfer or two-phase boiling heat transfer at high mass flux. This will eventually help us in designing effective design guidelines for supercritical boilers. Even inspiration from this can be drawn to explore other SF like methane which also experiences phenomena ${ }^{28}$ similar to HTD at supercritical pressure when used as a coolant in rocket engines.

Furthermore, the current literature lacks in terms of mathematical explanation for the decrease in the heat transfer of supercritical flow. Almost all previous works have mentioned property variations to be the reason for HTD. However, very few attempts have been made to quantify these variables. Typically boiling heat transfer is governed by the forces arising out of the density variation, so SF will experience a similar behavior. The same is explored for calculating the forces by first employing a parametric study of the various thermophysical properties. It reveals the key dominant property responsible for flow alteration. Subsequently, the actual flow is simplified for mathematical analysis based on the insight of the parametric study, and the same has been used to elucidate the observed flow characteristics.

Finally, we investigated the pseudoflow boiling characteristics by plotting the volume fraction data at the different axial locations for various flow conditions. This variable also provides us the distance of the two-phase interface from the wall, and the same has been nondimensionalized based on the scaling analysis. Further, the proposed scaling model leads to a theoretical equation that enables the prediction of the two-phase interface distance from the wall. This expression can provide a vital length scale for SF flow as it estimates the distance of the region near the wall where most of the properties vary drastically.

\section{METHODOLOGY}

\section{A. Governing equations}

This paper primarily covers the results obtained using the proposed two-phase method for the supercritical flow. However, some section of the paper (Secs. III A and IIIB) includes some results obtained using the conventional single-phase method. The inclusion is aimed for better qualitative perspective. Hence, for completeness, this section has two subparts that discuss the equations solved in both methods. All the flow governing equation of mass, momentum, and energy is numerically solved for the both the approach using commercial software (ANSYS FLUENT). The axis-symmetric flow for a steady-state case has been studied for the circular pipe flow. Accordingly, the governing equations have the radial and axial component, written in cylindrical coordinates for presentation.

Single-phase approach:

Continuity equation:

$$
\frac{1}{\mathrm{r}}\left\{\frac{\partial}{\partial \mathrm{x}}(\mathrm{r} \rho \mathrm{u})+\frac{\partial}{\partial \mathrm{r}}(\mathrm{r} \rho \mathrm{v})\right\}=0 .
$$

Axial momentum equation:

$$
\begin{aligned}
\frac{1}{\mathrm{r}}\left\{\frac{\partial}{\partial \mathrm{x}}\left(\mathrm{r} \rho \mathrm{u}^{2}\right)+\frac{\partial}{\partial \mathrm{r}}(\mathrm{r} \rho \mathrm{uv})\right\} \\
=-\frac{\partial \mathrm{p}}{\partial \mathrm{x}}-\rho \mathrm{g}+\frac{1}{\mathrm{r}} \frac{\partial}{\partial \mathrm{x}}\left\{2 \mathrm{r} \mu_{\mathrm{e}}\left(\frac{\partial \mathrm{u}}{\partial \mathrm{x}}-\frac{1}{3}(\nabla \cdot \overrightarrow{\mathrm{u}})\right)\right\} \\
\quad+\frac{1}{\mathrm{r}} \frac{\partial}{\partial \mathrm{r}}\left\{\mathrm{r} \mu_{\mathrm{e}}\left(\frac{\partial \mathrm{u}}{\partial \mathrm{r}}+\frac{\partial \mathrm{v}}{\partial \mathrm{x}}\right)\right\} .
\end{aligned}
$$

Radial momentum direction:

$$
\begin{aligned}
& \frac{1}{\mathrm{r}}\left\{\frac{\partial}{\partial \mathrm{x}}(\mathrm{r} \rho \mathrm{vu})+\frac{\partial}{\partial \mathrm{r}}\left(\mathrm{r} \rho \mathrm{v}^{2}\right)\right\} \\
& =-\frac{\partial \mathrm{p}}{\partial \mathrm{r}}+\frac{1}{\mathrm{r}} \frac{\partial}{\partial \mathrm{r}}\left\{2 \mathrm{r} \mu_{\mathrm{e}}\left(\frac{\partial \mathrm{v}}{\partial \mathrm{r}}-\frac{1}{3}(\nabla \cdot \overrightarrow{\mathrm{u}})\right)\right\} \\
& +\frac{1}{\mathrm{r}} \frac{\partial}{\partial \mathrm{x}}\left\{\mathrm{r} \mu_{\mathrm{e}}\left(\frac{\partial \mathrm{u}}{\partial \mathrm{r}}+\frac{\partial \mathrm{v}}{\partial \mathrm{x}}\right)\right\}-2 \mu \frac{\mathrm{v}}{\mathrm{r}^{2}}+\frac{2}{3} \frac{\mu}{\mathrm{r}}(\nabla \cdot \overrightarrow{\mathrm{u}}), \\
& \nabla \cdot \overrightarrow{\mathrm{u}}=\frac{\partial \mathrm{u}}{\partial \mathrm{x}}+\frac{\partial \mathrm{v}}{\partial \mathrm{r}}+\frac{\mathrm{v}}{\mathrm{r}} .
\end{aligned}
$$

Here, $\mathrm{x}$ and $\mathrm{r}$ are the axial distance from the inlet and radial distance from the axis, respectively, as shown in Fig. 4. Similarly, $\mathrm{u}$ and $\mathrm{v}$ are the axial and radial velocities correspondingly. Also, $\mu_{e}$ is the effective viscosity defined as $\mu_{e}=\mu$ (viscosity $)+\mu_{t}$, where $\mu_{t}$ is the turbulent viscosity to be determined by Eq. (5). Pressure and density are represented as $\mathrm{p}$ and $\rho$, respectively

$$
\mu_{t}=\rho C_{\mu} f_{\mu} \frac{k^{2}}{\varepsilon} .
$$

Here, $C_{\mu}$ is a constant and $f_{\mu}$ is a damping function to accommodate near wall effects.

Energy equation:

$$
\begin{aligned}
\frac{1}{\mathrm{r}}\left\{\frac{\partial}{\partial \mathrm{x}}(\mathrm{r} \rho \mathrm{uH})+\frac{\partial}{\partial \mathrm{r}}(\mathrm{r} \rho \mathrm{vH})\right\} \\
=\frac{1}{\mathrm{r}}\left\{\frac{\partial}{\partial \mathrm{x}}\left[\mathrm{rC}_{\mathrm{p}}\left(\frac{\mu}{\operatorname{Pr}}+\frac{\mu_{\mathrm{t}}}{\operatorname{Pr}_{\mathrm{t}}}\right) \frac{\partial \mathrm{T}}{\partial \mathrm{x}}\right]+\frac{\partial}{\partial \mathrm{r}}\left[\mathrm{rC}_{\mathrm{p}}\left(\frac{\mu}{\operatorname{Pr}}+\frac{\mu_{\mathrm{t}}}{\operatorname{Pr}_{\mathrm{t}}}\right) \frac{\partial \mathrm{T}}{\partial \mathrm{r}}\right]\right\} \\
+D_{\text {VISC. }}
\end{aligned}
$$

Here, $\mathrm{H}$ stands for the specific enthalpy, $\mathrm{T}$ is the temperature, $D_{\text {VISC }}$ is the viscous dissipation term, and $C_{p}$ represents the specific heat at constant pressure. $P r$ is the Prandtl number whereas $\operatorname{Pr}_{t}$ represents the turbulent Prandtl number, which is equal to 0.85 for this analysis.

The shear stress transport (SST) $\mathrm{k}-\omega$ turbulence model is utilized here, which has the advantage that it subsumes the qualities of the $\mathrm{k}-\omega$ and the $\mathrm{k}-\varepsilon$ model. It has the properties of $\mathrm{k}-\omega$ in-wall proximity while acting like $\mathrm{k}-\varepsilon$ in the free stream region. The governing equations of the turbulence model are shown below for turbulent kinetic energy $(\mathrm{k})$ (TKE) and turbulence dissipation rate $(\varepsilon)$,

$$
\begin{aligned}
& \left\{\frac{\partial}{\partial \mathrm{x}}(\rho \mathrm{uk})+\frac{1}{r} \frac{\partial}{\partial \mathrm{r}}(\mathrm{r} \rho \mathrm{vk})\right\} \\
& =\frac{\partial}{\partial \mathrm{x}}\left[\left(\mu+\frac{\mu_{\mathrm{t}}}{\operatorname{Pr}_{\mathrm{k}}}\right) \frac{\partial \mathrm{k}}{\partial \mathrm{x}}\right]+\frac{1}{r} \frac{\partial}{\partial \mathrm{r}}\left[r\left(\mu+\frac{\mu_{\mathrm{t}}}{\operatorname{Pr}_{\mathrm{k}}}\right) \frac{\partial \mathrm{k}}{\partial \mathrm{r}}\right] \\
& \quad+P_{k}+G_{k}-\rho \varepsilon,
\end{aligned}
$$




$$
\begin{aligned}
& \left\{\frac{\partial}{\partial \mathrm{x}}(\rho \mathrm{u} \varepsilon)+\frac{1}{r} \frac{\partial}{\partial \mathrm{r}}(\mathrm{r} \rho \mathrm{v} \varepsilon)\right\} \\
& =\frac{\partial}{\partial \mathrm{x}}\left[\left(\mu+\frac{\mu_{\mathrm{t}}}{\operatorname{Pr}_{\varepsilon}}\right) \frac{\partial \mathrm{k}}{\partial \mathrm{x}}\right]+\frac{1}{r} \frac{\partial}{\partial \mathrm{r}}\left[r\left(\mu+\frac{\mu_{\mathrm{t}}}{\operatorname{Pr}_{\varepsilon}}\right) \frac{\partial \mathrm{k}}{\partial \mathrm{r}}\right] \\
& +C_{\varepsilon 1} f_{1} \frac{\varepsilon}{k}\left(P_{k}+G_{k}\right)-C_{\varepsilon 2} f_{2} \rho \frac{\varepsilon^{2}}{k}+\rho E .
\end{aligned}
$$

Here, $P_{k}$ and $G_{k}$ are the turbulence production term due to mean velocity and buoyancy, respectively. The expression for the same is given in Eq. (9). Also, $C_{\varepsilon 1}$ and $C_{\varepsilon 2}$ are the model constants, whereas $f_{1}$, $f_{2}$, and $E$ are the damping functions. $\operatorname{Pr}_{\varepsilon}$ and $\operatorname{Pr}_{\mathrm{k}}$ are the turbulent Prandtl number corresponding to $\mathrm{k}$ and $\varepsilon$

$$
\begin{gathered}
P_{k}=\mu_{\mathrm{t}}\left[2\left\{\left(\frac{\partial u}{\partial x}\right)^{2}+\left(\frac{\partial v}{\partial r}\right)^{2}+\left(\frac{v}{r}\right)^{2}\right\}+\left(\frac{\partial u}{\partial r}+\frac{\partial v}{\partial x}\right)^{2}\right] \\
G_{k}=-\beta g \frac{\mu_{\mathrm{t}}}{\operatorname{Pr}_{\mathrm{t}}} \frac{\partial T}{\partial X_{i}} .
\end{gathered}
$$

Here, $\beta$ is the coefficient of thermal expansion and $X_{i}$ denotes the $i$ th coordinate used in the flow analysis.

Two-phase approach:

The volume of fluid (VOF) multiphase model has been employed for the two-phase simulation. The VOF model is generally used to track the interfaces between immiscible phases by calculating the volume fractions of all phases in each computational cell. However, with the recent developments, ${ }^{29-31}$ VOF has proven its effectiveness for the flow boiling process, which involves mass transfer across the phases. In VOF model, the continuity equation is solved individually for the volume fraction $(\alpha)$ of the second phase, which is vapor $\left(\alpha_{\mathrm{v}}\right)$ in the present case. Hence, the equation takes the following form:

$$
\frac{1}{\rho_{\mathrm{v}}}\left[\frac{\partial\left(\alpha_{\mathrm{v}} \rho_{\mathrm{v}}\right)}{\partial \mathrm{t}}+\nabla\left(\alpha_{\mathrm{v}} \dot{\rho}_{\mathrm{v}} \overrightarrow{\mathrm{u}}_{\mathrm{v}}\right)=\left(\dot{\mathrm{m}}_{\mathrm{lv}}-\dot{\mathrm{m}}_{\mathrm{vl}}\right)\right] .
$$

Here, $\dot{\mathrm{m}}_{\mathrm{vl}}$ and $\dot{\mathrm{m}}_{\mathrm{lv}}$ represent the mass transfer rates from vapor to liquid and liquid to vapor, respectively, and both are governed by the Lee model. ${ }^{32}$ The expression for both the mass transfer rates is given in the following equation:

$$
\begin{gathered}
\text { If } T_{l}(\text { liquid } T)>T_{\text {sat }} \\
\dot{\mathrm{m}}_{\mathrm{lv}}=f_{e} \alpha_{l} \rho_{l} \frac{\left(T_{l}-T_{\text {sat }}\right)}{T_{\text {sat }}} \\
\text { If } T_{v}(\text { liquid } T)<T_{\text {sat }} \\
\dot{\mathrm{m}}_{\mathrm{vl}}=f_{c} \alpha_{v} \rho_{v} \frac{\left(T_{\text {sat }}-T_{v}\right)}{T_{\text {sat }}}
\end{gathered}
$$

Here, $f_{e}$ and $f_{c}$ are the evaporation and condensation frequency, respectively. $T_{\text {sat }}$ is the saturation temperature, which is assumed to be equal to the pseudocritical temperature $\left(T_{p c}\right)$ in the proposed twophase method. The continuity equation for the second phase is only solved, and the volume fraction for the primary phase (liquid) or $\alpha_{1}$ is calculated subject to the constraint shown below

$$
\alpha_{1}+\alpha_{\mathrm{v}}=1
$$

All the other governing equations, such as momentum, energy, and turbulence model, are solved for the fluid as a whole [same as single fluid equations which are given earlier equations from (2) to (9)]. All the properties must be taken as volume averaged, having a contribution from every phase present in the computational cell. In the other words, there is a single momentum, turbulence and an energy equation for the multiphase flow with modified properties accounting for the presence of different phases. Consequently, all the flow variables computed from the governing equation incorporates the existence of all the phases.

Further, the energy required for the phase transition is accommodated by setting a common reference temperature for both the phases during enthalpy. In other words, phase-wise enthalpies are measured with a jump while changing the phase as manifested in Fig. 3. For the present study, pseudocritical temperature $\left(\mathrm{T}_{\mathrm{pc}}\right)$ is assumed to be the saturation temperature $\left(\mathrm{T}_{\mathrm{sat}}\right)$ for the pseudophase change model, and the same has been kept as the reference temperature. Hence, to incorporate the phase change process, the enthalpy of the LSCW $\left(\mathrm{H}_{1}^{\text {sat }}\right)$ and VSCW $\left(\mathrm{H}_{\mathrm{v}}^{\text {sat }}\right)$ at the reference temperature is fixed as zero and $\mathrm{H}_{\text {trans, }}$, respectively. Figure 3 depicts how the pseudophase change model incorporates
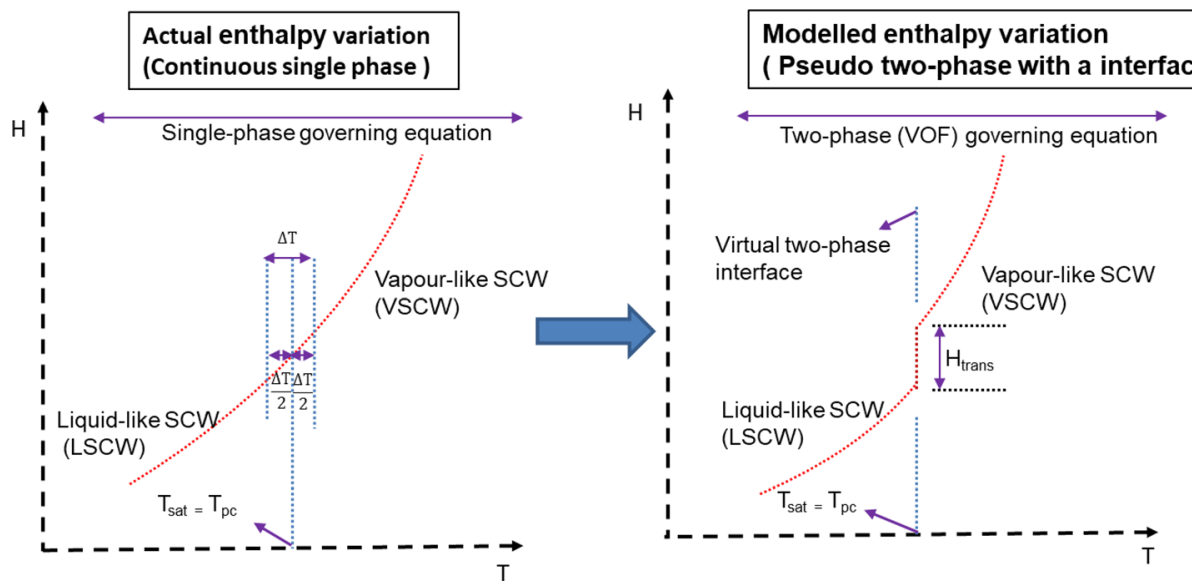

FIG. 3. A Schematic representing the actual and pseudoenthalpy variation. The left side of the figure depicts the real enthalpy variation of SCW, which has a monotonous increase in its value with temperature. Although the right side of the figure maintains the same variation with temperature, there is a sudden jump in enthalpy value at $T_{p c}$. So, the juxtaposition of the two images reveals how the inspiration has been drawn from the subcritical phase change process, and the same has been incorporated. 
the phase change energy requirements in the form of $\mathrm{H}_{\text {trans }}$ such that it satisfies the following equation:

$$
\mathrm{H}_{\mathrm{v}}^{\text {sat }}-\mathrm{H}_{1}^{\text {sat }}=\mathrm{H}_{\text {trans }} .
$$

$\mathrm{H}_{\text {trans }}$ is governed by $\Delta \mathrm{T}$ and average specific heat $\left(\overline{\mathrm{C}_{\mathrm{p}}}\right)$ in the $\Delta \mathrm{T}$ temperature range. As displayed in Figs. 1 and $3, \Delta \mathrm{T}$ is the temperature interval in the pseudocritical region used to replicate a pseudo-twophase saturation interface. Therefore, $\Delta \mathrm{T}$ controls the extent of the energy required for the phase transition $\left(\mathrm{H}_{\text {trans }}\right)$ across this two-phase boundary. The value of $\mathrm{H}_{\text {trans }}$ at a given pressure can be computed by the basic definition of enthalpy given in the following equation:

$$
\mathrm{H}_{\text {trans }}=\overline{\mathrm{C}_{\mathrm{p}}}(\Delta \mathrm{T}) \text {. }
$$

Further, all the properties experience a jump in their values in a similar manner as enthalpy shown in Fig. 3. Hence, once the fluid temperature crosses $\mathrm{T}_{\mathrm{sat}}=\mathrm{T}_{\mathrm{pc}}$, a transition of LSCW to VSCW happens, and the same is reflected in the fluid properties. The difference in density (or other properties) of VSCW to LSCW is dictated by the $\Delta \mathrm{T}$ value since this value controls how the density at the saturation temperature is defined for the two phases. The expression for the same is given in Eq. (15), which shows that LSCW has a density value of SCW density corresponding to a temperature of $\left(T_{p c}-\frac{\Delta T}{2}\right)$ whereas for VSCW it is $\left(T_{p c}+\frac{\Delta T}{2}\right)$

$$
\rho_{\mathrm{LSCW} \text { at } T_{s a t}}=\rho_{T_{p c}-\frac{\Delta T}{2}} \text { and } \rho_{\mathrm{VSCW} \text { at } T_{s a t}}=\rho_{T_{p c}+\frac{\Delta T}{2}} \text {. }
$$

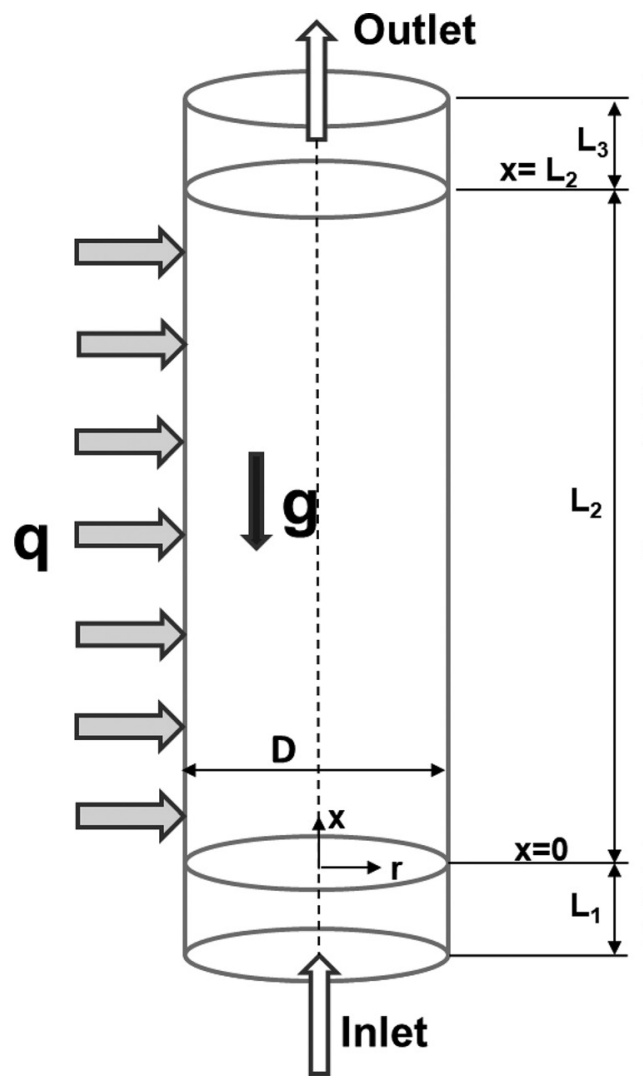

Here, the property transition incorporated in the pseudophase change model is demonstrated with the help of density; however, it occurs in a similar fashion for all the other properties. It is worth mentioning that this pseudophase change model behaves like any typical subcritical phase change process, where the saturation zone (two-phase zone) is formed. The temperature variation is continuous as displayed in Fig. 3 with the fluid experiencing change in properties across the two-phase region.

\section{B. Physical and numerical model}

Figure 4 depicts the general representation of the computational domain where the circular pipe's length and diameter vary depending on the experiments, as shown in Table I. The flow geometry used is a vertical circular pipe; for numerical analysis purpose, it is partitioned into three different regions along the axis. The first part is a region where no heat transfer occurs and results in a developing velocity profile overcoming the entrance effects (region 1). The next region imitates the heated length (uniform heat flux) of the test section in various experimental setups (region 2) as shown in Fig. 4. In the end, a region with an adiabatic wall ensures no backward flow, leading to a stable numerical system (region 3).

No-slip condition is maintained near the wall. As previous works suggest, the nondimensional wall distance $\left(y^{+}\right)$of the first element near the wall should be less than 1 . This has been accommodated by introducing radial nonuniformity in the mesh such that the highest
Region 2 (Heated):

a. Uniform heat Flux at the wall

b. Developed velocity profile (independent of entrance effect)

c. Uniform Bulk Temperature at the entry

\section{Region 1(Unheated):}

a. Adiabatic wall

b. Uniform Bulk Temperature at the entry
FIG. 4. Schematic diagram of flow geometry used for the simulation purpose. Although the figure represents a threedimensional computation domain, an axissymmetric coordinates system has been used for the numerical study. Also, region 2 mimics the actual experimental test condition in terms of diameter, length, alignment, and boundary condition. The other two regions are used either to achieve the desired boundary condition or for convenience in the numerical study. 
TABLE I. Geometric specification for the computational domain.

\begin{tabular}{|c|c|c|c|c|c|}
\hline Geometry & $\begin{array}{c}\text { Cross } \\
\text { section }(\mathrm{D})(\mathrm{mm})\end{array}$ & $\begin{array}{c}\text { Unheated } \\
\text { Length }\left(\mathrm{L}_{1}\right)(\mathrm{mm})\end{array}$ & $\begin{array}{c}\text { Heated } \\
\text { length }\left(\mathrm{L}_{2}\right)(\mathrm{mm})\end{array}$ & $\begin{array}{c}\text { Unheated } \\
\text { length }\left(\mathrm{L}_{3}\right)(\mathrm{mm})\end{array}$ & $\begin{array}{c}\text { Total } \\
\text { length }(\mathrm{mm})\end{array}$ \\
\hline Circular (Shitsman) & 8 & 100 & 1500 & 100 & 1700 \\
\hline Circular (Ornatskij) & 3 & 100 & 800 & 100 & 1000 \\
\hline
\end{tabular}

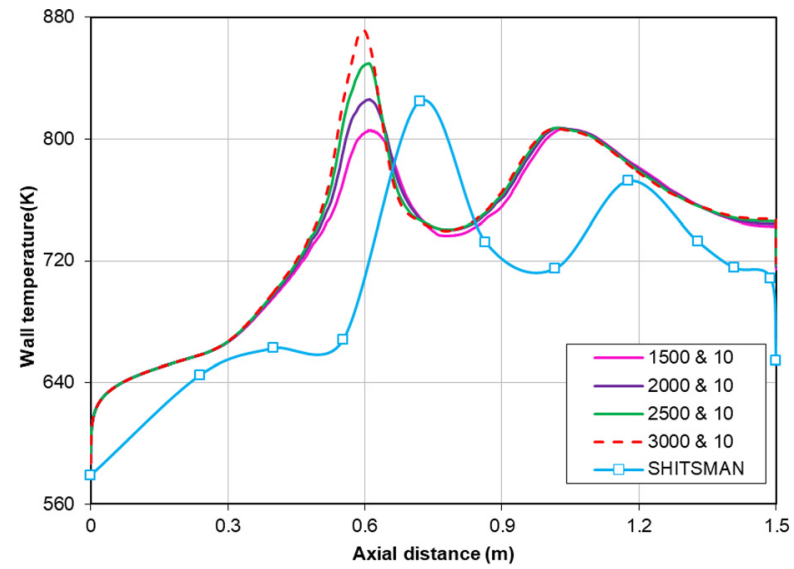

FIG. 5. Wall temperature variation for different pair of $f_{e}$ and $f_{c}$.

grid density exists in the wall neighborhood. The distance of the first grid from the wall is kept at $0.001 \mathrm{~mm}$ to maintain the value of $\mathrm{y}^{+}$less than 0.2 so that it falls within the recommended limit.

The property variation across the pseudocritical line resembles the phase-change process in the subcritical fluid, as shown in Fig. 1. To set the analogy with conventional boiling process, a virtual phasechange process across the pseudocritical line is assumed and the same is approached using the VOF model. The phase transition is implemented by the mass transfer mechanism according to the Lee model. It is a condensation-evaporation model with a user-defined coefficient (or frequency) with no specific guideline for selecting the same. The conventional method is to do the simulations for different coefficient values and select the pair that emulates the experimental results.
In the present study, Shitsman ${ }^{5}$ experimental result (case-1) was taken as reference, and the traditional approach was followed, as shown in Fig. 5. The pair was selected based on the following reasons: it predicted two peaks, and the difference between two maxima temperatures $(\sim 50 \mathrm{~K})$ is similar to experimental data. Accordingly, the value of $f_{e}$ and $f_{c}$ was chosen to be equal to 2500 and 10 , respectively, and the same values of frequency have been incorporated for the simulation.

The surface tension effects are assumed to be insignificant in the proposed method because of the similarity of the supercritical fluid to a second-order phase transition. In the second-order transition, the variation in the properties is continuous, unlike first-order phase transition, where there is a sudden jump in the properties of two phases present. Therefore, for following the actual flow, $\Delta \mathrm{T}$ should be the least. In the present setup, $2 \mathrm{~K}$ is the minimum that can be applied. This is supported by the fact that Ansys Fluent limits the number of data points which can be used for a piecewise linear property profile. The same has been employed to incorporate the properties as a function of temperature, assuming pressure to be constant. This is a valid assumption since the pressure drop is significantly less.

In order to accommodate a temperature range subsuming all the possible flow temperatures, we have maintained a difference of $1 \mathrm{~K}$ between two subsequent temperature data points in the pseudocritical region. This eventually leads to the least value of $2 \mathrm{~K}$ for $\Delta \mathrm{T}$ because of the symmetric distribution over the pseudocritical temperature. However, it is essential to point out that there is no prescribed method of choosing $\Delta \mathrm{T}$ and one is free to use the least value possible; this introduces a default parameter in the model.

National Institute of Standards and Technology (NIST) software miniREFPROP has been used for thermophysical properties of water at various operating conditions. The geometric details for both cases are given in Table I.

TABLE II. Boundary and operating condition.

\begin{tabular}{lccc}
\hline \hline Parameter & Case-1 & Case-2 & Case-3 \\
\hline Reference & $\begin{array}{c}\text { Shitsman experimental } \\
\text { setup with HTD having two } \\
\text { peaks in the wall temperature }\end{array}$ & $\begin{array}{c}\text { Ornatskij (1971) experimental } \\
\text { setup with HTD }\end{array}$ & $\begin{array}{c}\text { Shitsman experimental } \\
\text { setup with no HTD }\end{array}$ \\
$\mathrm{T}_{\mathrm{pc}}$ (Pseudocritical temperature) $(\mathrm{K})$ & 659 & & 659 \\
$\bar{G}$ (Mass flow rate) (kg/s) & 0.022 & 0.0106 & 652 \\
$\mathrm{~T}_{\text {in }}$ (Inlet temperature) $(\mathrm{K})$ & 578 & 500 & 0.022 \\
$\mathrm{q}\left(\right.$ Heat flux) $\left(\mathrm{kW} / \mathrm{m}^{2}\right)$ & 384.8 & 1810 & 600 \\
$\mathrm{P}_{\text {out }}$ (Outlet pressure) $(\mathrm{MPa})$ & 25.3 & 25.3 & 220.8 \\
$\mathrm{Re}_{\text {in }}$ (Inlet Reynolds number) & 38885 & 36958 & 23.3 \\
$\mathrm{Bo}^{*}$ (Inlet buoyancy parameter) & $1.039 \times 10^{-6}$ & $3.8865 \times 10^{-8}$ & 43129 \\
\hline \hline
\end{tabular}




\section{Boundary and operating conditions}

The boundary and operating conditions are given in Table II for all the cases. The flow conditions are taken from Shitsman ${ }^{5}$ and Wen ${ }^{19}$ experiments, where HTD has been imputed to buoyancy and

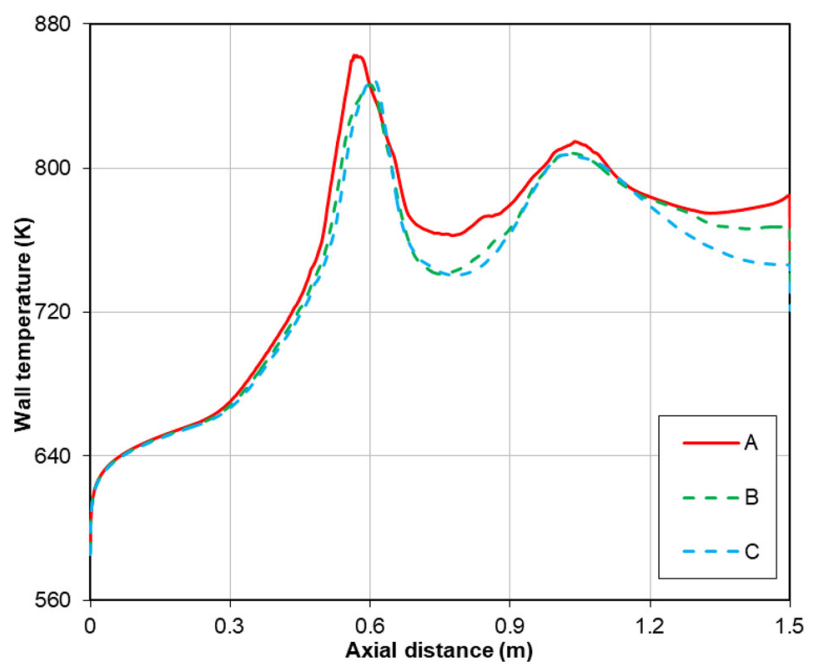

(a) $\mathrm{A}=200000$ nodes, $\mathrm{B}=2 \mathrm{~A}$ and $\mathrm{C}=2 \mathrm{~B}$

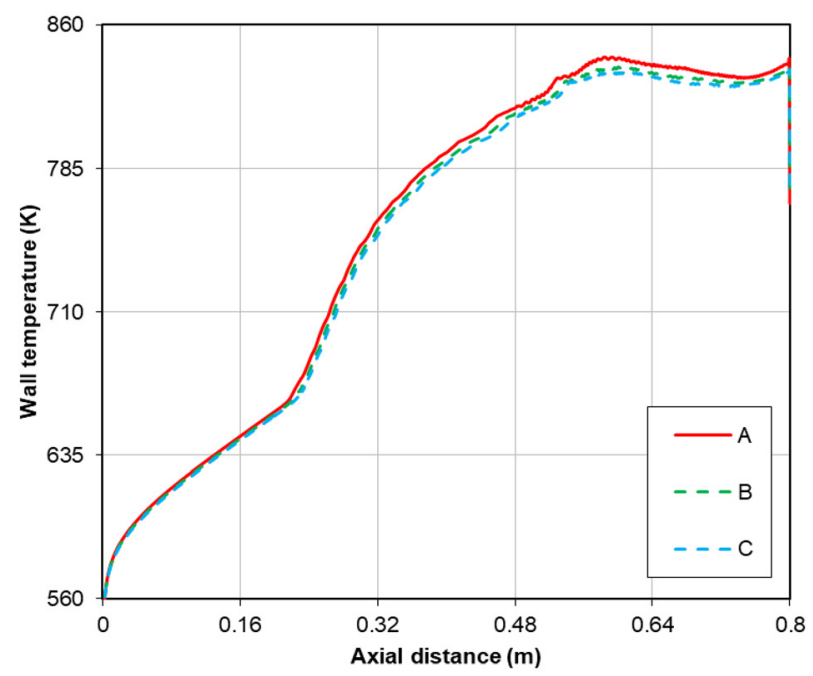

(b) $\mathrm{A}=60000$ nodes, $\mathrm{B}=2 \mathrm{~A}$ and $\mathrm{C}=2 \mathrm{~B}$

FIG. 6. (a) and (b) show the wall temperature variation for case-1 and case-2 during the grid independence study for the two-phase approach. The plot reveals that the result achieves grid independence at B number of nodes for both the cases. Also, it has been noticed that the wall temperature is insensitive to the grid refinement in the axial direction. Based on the above observation, the final mesh structure was decided to balance accuracy and computational cost. So, all the simulations are done on the grid structure with the specification are axial element size $(\mathrm{dx})=0.6 \mathrm{~mm}$ and radial element size $(\mathrm{dr})=0.03 \mathrm{~mm}$ for both the cases, leading to 447000 nodes and 123000 nodes for case- 1 and case-2, respectively. acceleration effects, respectively. At the inlet boundary, turbulent intensity and turbulent viscosity ratio are $5 \%$ and $10 \%$, respectively. Since the fluid is compressible, mass flow inlet and pressure outlet have been applied as the boundary conditions. The gravitational acceleration $(\mathrm{g})$ value is taken to be $9.8 \mathrm{~m} / \mathrm{s}^{2}$. Reynolds number $(\mathrm{Re})$, Grashof number (Gr), Prandtl number (Pr), and buoyancy parameter (Bo*) are defined below in Eq. (16), and the same has been calculated based on the inlet bulk properties

$$
\operatorname{Re}=\frac{\rho U D}{\mu}, \quad G r=\frac{\beta g D^{4} q \rho^{2}}{\lambda \mu^{2}}, \quad \operatorname{Pr}=\frac{\mu C_{p}}{\lambda} \& B o^{*}=\frac{G r}{R e^{3.425} \operatorname{Pr}^{0.8}} .
$$

Here, $\lambda$ is the thermal conductivity.

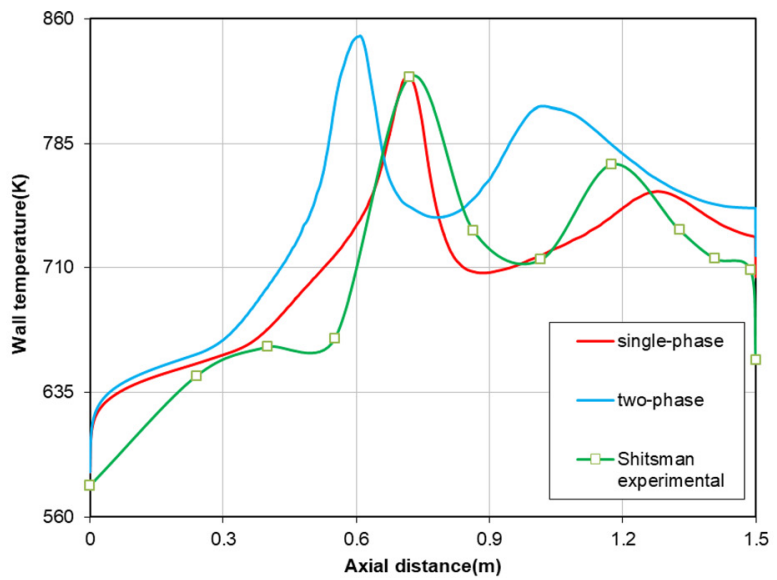

(a)

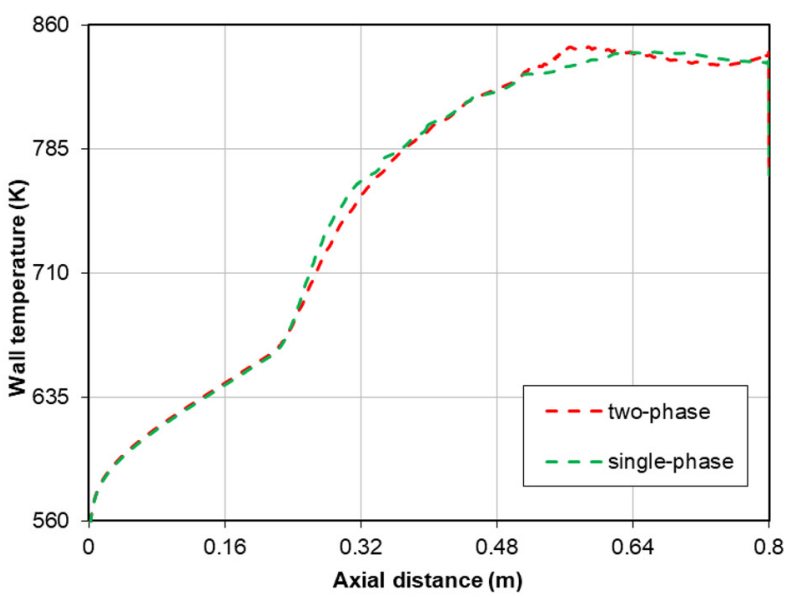

(b)

FIG. 7. Wall temperature axial (streamwise) variations: (a) In case-1, Shitsman's experimental data have been interpolated to get a curve, and the same has been used for the comparison with the numerical result. (b) Case-2, the original Ornatskij's data were not available, but the wall temperature profile has been checked against the reported data in the literature ${ }^{19}$ and follows the same trend. 


\section{RESULTS AND DISCUSSION}

\section{A. Grid independence}

All the simulation results have been ensured to be gridindependent and the same has been manifested for two-phase method in Figs. 6(a) and 6(b). Mesh has been refined in radial as well as in the axial direction. Initially, the number of nodes was kept at 200000 and 60000 for case-1 and case-2, respectively; subsequently, the grid was modified. Although the mesh is refined radially, the distance of the first grid point near the wall is kept the same in all the simulations to maintain the desired $\mathrm{y}^{+}$ value.

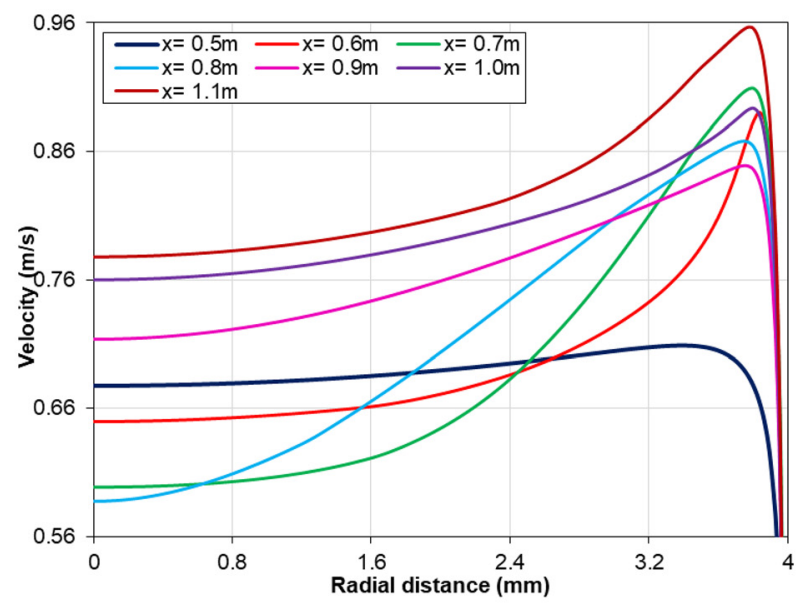

(a)

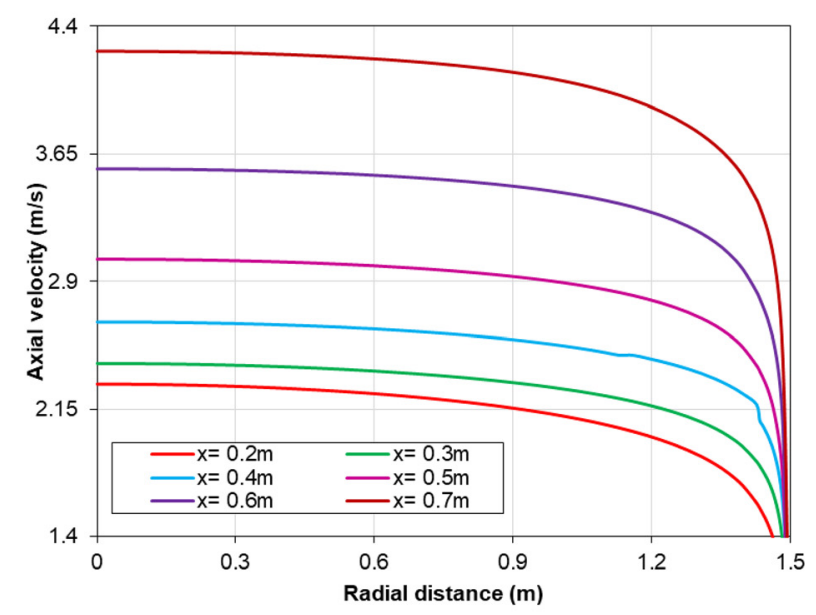

(b)

FIG. 8. Radial variation of the velocity profile at different axial locations: (a) Case-1 suggests that the major manipulation of the flow field is shifting from the near the wall region to the bulk fluid downstream. (b) Case-2, here contrary to the first case, the alteration of the flow field is concentrated in the bulk region throughout the flow domain. Also, the magnitude of velocity is one order higher than the case-1 owing to higher mass flux for case-2.

\section{B. Comparison with the experimental result and conventional (single-phase) method}

The two-phase model result is compared against the experimental results ${ }^{5,19}$ as well as the single-phase method to check the extent of suitability. The inclusion of the single-phase result is to provide a qualitative perspective of the proposed two-phase method by contrasting it with the conventional single-phase approach. Also, to establish a comprehensive application of the proposed model, case- 1 and case- 2 have been studied. Although both cases correspond to the HTD phenomena, the source responsible for the impairment of heat transfer is very different. Figures 7(a) and 7(b) exhibit a comparison of numerical and

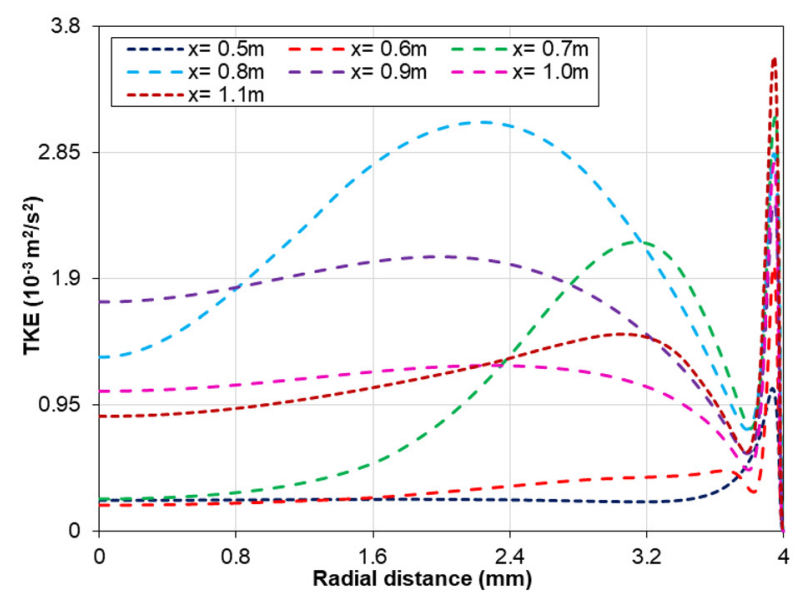

(a)

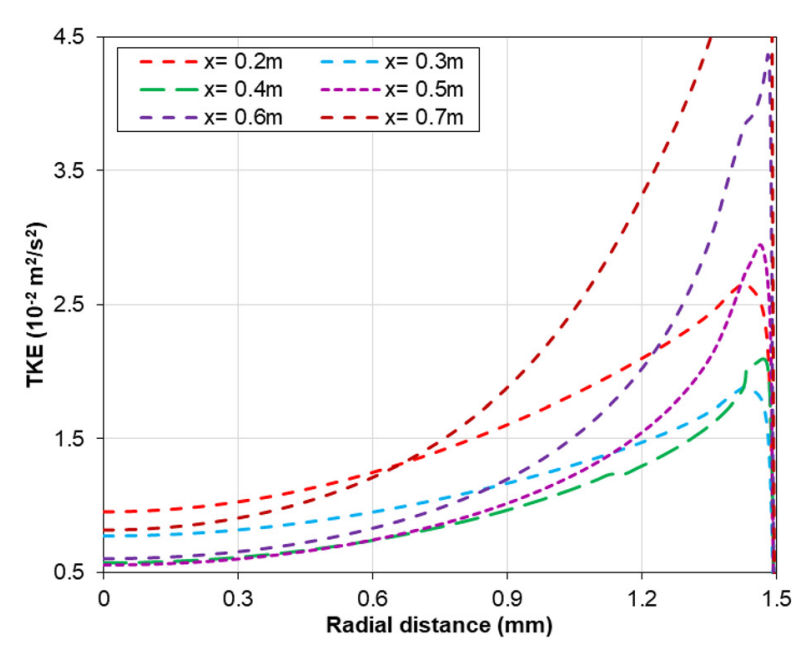

(b)

FIG. 9. Radial TKE profile at the different axial locations: (a) Case-1, here decline in TKE value is evident in near the wall region or in the bulk depending on the axial position. (b) In case-2, the loss in TKE is accumulated in the bulk region despite the increase in the TKE near the wall. Similar to velocity magnitude, the TKE values are higher than one order for case-2 compared to case-1. 
experimental results for both cases. The juxtaposition reveals that numerical analysis is competent enough to predict the right trend and reveals the presence of pseudoflow boiling in SF flow. However, there are inconsistencies in the magnitude and the location of the HTD as symbolized by the wall temperature peaks.

It is a well-established fact that any turbulence loss directly results in downgrading the convective heat transfer, which largely depends on fluid mixing. So, for HTD to happen, there must be a plunge in the TKE, which is the best evaluating parameter for the flow turbulence. Besides, these forces originating out of the fluid property disparity alter the flow, which is reflected in the velocity plots. The slope of the velocity profile qualifies to be the best analysis parameter for TKE. All this leads to the conclusion that any decrease in the velocity gradient will be reflected as a decline in the TKE value.

Figure 7(a) depicts case-1, which corresponds to Shitsman experimental setup, ${ }^{5}$ where the two wall temperature peaks were observed. Peculiarly HTD reoccurred after showing signs of recovery. The numerical study matches the observed trend, although the two-phase and single-phase approaches do not imitate each other perfectly. However, this can be anticipated because the VOF model and the pseudophase change process have a lot of parameters that need to be properly fine-tuned. In the vicinity of the first peak, $\mathrm{x}=0.5 \mathrm{~m}$ velocity
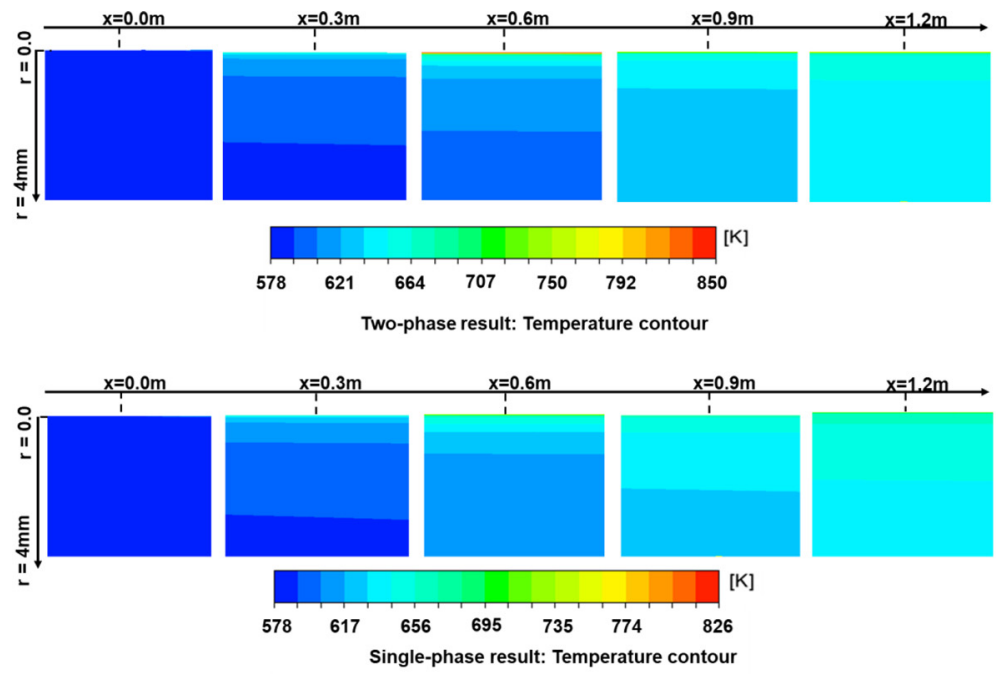

(a)

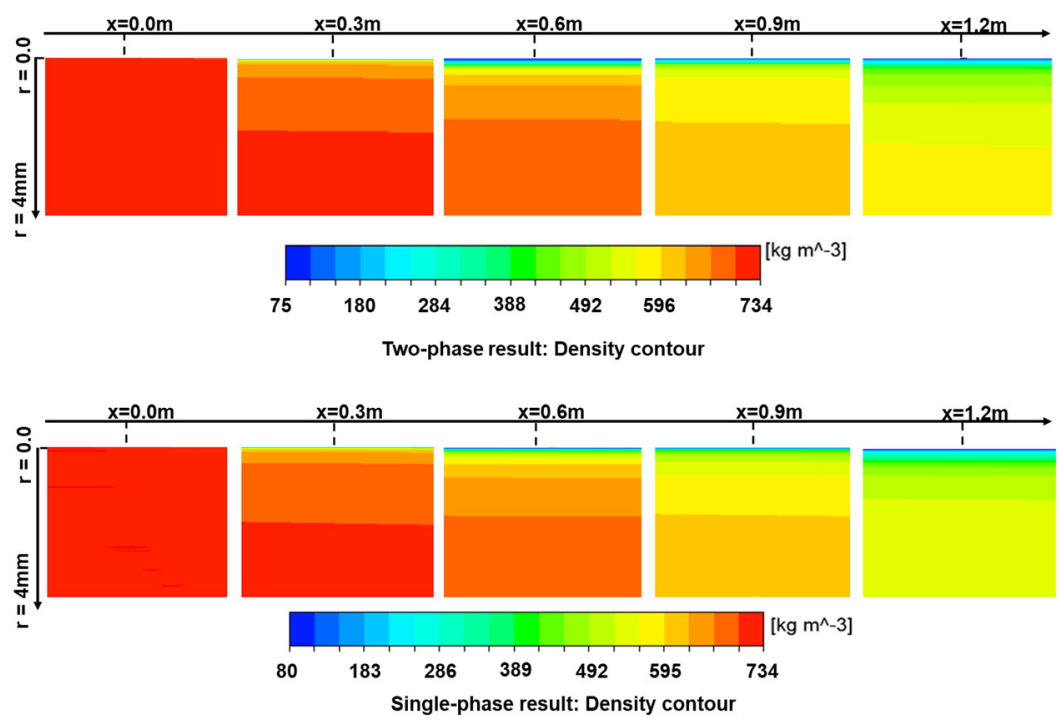

FIG. 10. Contour comparison of the results from two-phase and single-phase approach for case-1. All the contours are in $x-r$ plane, so that horizontal direction and vertical direction are along the $x$ axis and $r$-axis, respectively. The value of $x$ at the top of each contour marks the axial location of the contour in the flow domain and the value of $r$ shows radial variation from the flow axis: (a) Temperature contour and (b) density contour.

(b) 
profile is flattened near the wall, as shown in Fig. 8(a) because of buoyancy forces arising out of the radial density variation. Meanwhile, as expected TKE values experience a drop near the first peak, displayed in Fig. 9(a). As the heat transfer recovery starts from the first peak location, the velocity profile suggests that buoyancy forces are still dominant in the near-wall region, eventually leading to higher velocity near the wall. This is also translated in the TKE plot, where the value keeps on increasing near the wall.

Further downstream, the flow experiences an increase in the velocity of the bulk fluid for $\mathrm{x}=0.9 \mathrm{~m}$ shown in Fig. 8(a), and this results in flattening of the velocity profile away from the wall and in the proximity of the second wall temperature peak. It leads to loss of TKE majorly in the bulk region at $\mathrm{x}=0.9 \mathrm{~m}$ and $\mathrm{x}=1.0 \mathrm{~m}$ as represented in Fig. 9(a), which eventually results in HTD. The reoccurrence of the heat transfer impairment is primarily due to the acceleration effects where the bulk fluid rushes to maintain the same mass flow rate despite the decreasing value of density in the axial direction.

Also for case-2 (which was inspired by the Ornatskij experiment ${ }^{19}$ ), Fig. 7(b) represents the two numerical approaches that produce very similar results. Contrary to case-1, mass flux and heat flux are much higher in this analysis; thus, flow dynamics is much different even though the fluid has the same property. This study falls into the category of HTD, which is solely the consequence of the acceleration effect. The rapid decrease in the fluid density propels the flow, as displayed in Fig. 8(b), where the velocity keeps on increasing downstream. As a result, the velocity gradient decreases in bulk, marked by the decline in TKE value even though it grows near the wall, as manifested in Fig. 9(b). Hence, a wall temperature peak is observed, which is figurative of HTD. Although the TKE rises near the wall, as shown in Fig. 9(b), it cannot compensate for the decrease in TKE. Since the heat transfer involves convection from the wall to bulk, any loss of turbulence at any radial location, will obstruct the heat flow.

In addition, Fig. 10 depicts the temperature and density contour plot for case- 1 for the proposed two-phase approach and conventional single-phase method. The plot shows that due to the formation of the saturation zone (two-phase region), the temperature in the bulk of flow is less in the case of the two-phase method compared to the single-phase result. It should be noted that two-phase region will absorb energy for the phase change process resulting in constant temperature, which is absent in the single-phase model. The same trend is experienced in the density contour as it decreases monotonically with temperature.

Further, the wall temperature maxima characteristic correlates with the nature of the forces responsible for the HTD. In other words, the temperature peak's nature is dictated by the way the altered flow field presents a barrier to heat transfer in the flow, and one can even draw an analogy to the thermal resistance circuit model used in the conduction problem. The two extremes of the temperature peaks are marked: the sharp ones resulting from buoyancy forces and broad ones are the consequence of the acceleration effects. The buoyancy results in heat transfer obstruction near the wall region, whereas the acceleration effects bar the heat transfer in the bulk fluid.

\section{Parametric study of properties}

The main objective of the parametric study is to unearth the most dominant property of the four fluid properties affecting the flow behavior. For this purpose, four simulations corresponding to each property have been performed using the conventional numerical setup (single-phase), keeping everything unchanged except the properties shown in Figs. 11(a) and 11(b). For each run, one of the properties is varied; meanwhile, the rest are maintained constant at their inlet value.

Figure 11(a) corresponds to case-1, which represents that the density variation has the most significant influence on the flow dynamics followed by the thermal conductivity, whereas specific heat has the least impact. Interestingly, the variable density simulation also predicts two wall temperature peaks which are already confirmed in Sec. III B.

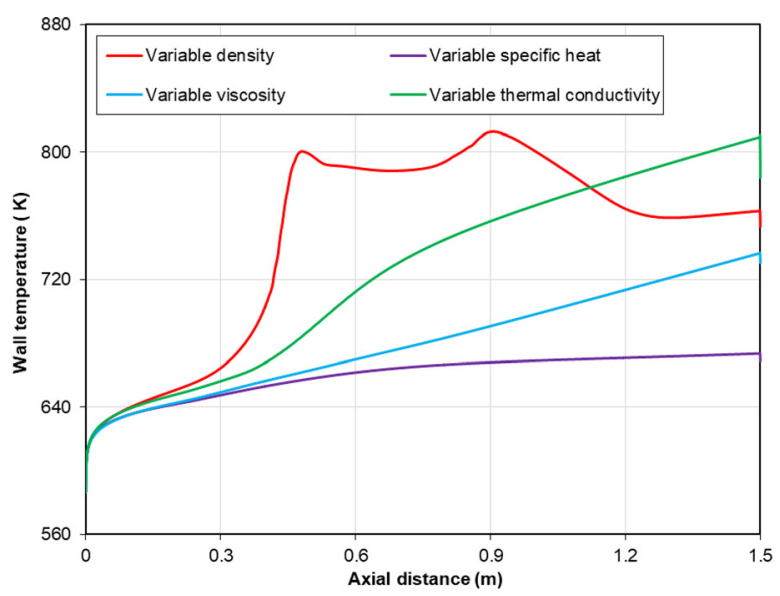

(a) .

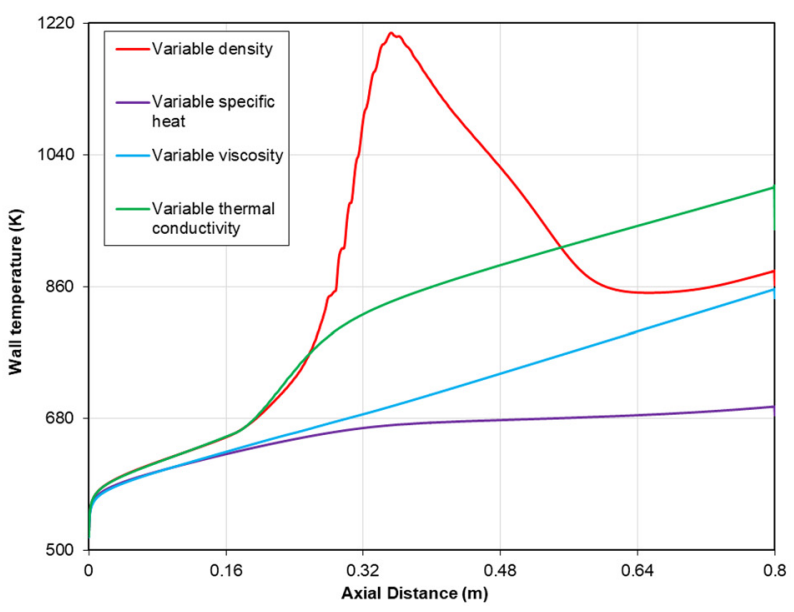

(b)

FIG. 11. Plots are depicting the parametric study of properties. (a) Case-1, the capability of variable density setup to predict the two peaks in wall temperature similar to actual flow reveals the extent to which density variation governs the SCW flow. (b) Case-2, here identical to the first case, density controls the flow field. Besides, the temperature peak is higher in magnitude and sharp compared to case-1 because of the lower cross-sectional area (CA) and higher heat flux than case-1. 
Similarly, Fig. 11(b) represents that for case-2, density difference only can forecast the HTD. However, the properties governing the flow characteristic follows the same order as observed in the case-1. This observation falls in line with prior understanding about the supercritical flow as all the forces altering the flow field, such as buoyancy or the acceleration effects, are direct consequences of the density disparity.

Further, the dominance of density variation points to the similarity of the SF flow with pseudoboiling (which has been already established in Sec. III B) as the forces arising out of the density variation govern a typical flow boiling phenomena. Besides, it is worth mentioning that with the different values of the constant properties instead of their inlet value, results may differ where the HTD may not be as prominent as it is in this study. All those possible analyses will suggest that the density has the highest control over the flow pattern.

\section{Quantitative analysis of flow}

The quantitative analysis aims to gauge the different forces numerally originating from the density difference in the axial and radial direction. As mentioned in the preceding sections, buoyancy and acceleration effects are the two broad classifications of forces responsible for the heat transfer impairment in supercritical flows. So, the actual flow has been simplified to calculate the magnitude of the two forces on a small axial element of $\Delta \mathrm{x}=0.01 \mathrm{~m}$ as represented in Fig. 12 based on following reasoning:

(1) The major share of the buoyant force is experienced in the near-wall region, where the flow has the highest radial density gradient. As displayed in Fig. 12, $\Delta \mathrm{r}$ is the cross-sectional area where the buoyant forces are predominant. Therefore, $\Delta \mathrm{r}$ is estimated based on the following equation:

$$
\left(\rho_{r-\Delta r}-\rho_{w}\right)=0.9\left(\rho_{c}-\rho_{w}\right),
$$

where $\rho_{\mathrm{c}}=$ density at the center, $\rho_{\mathrm{w}}=$ density at the wall, and $\rho_{\mathrm{r}-\Delta \mathrm{r}}=$ density at a radial distance of $\mathrm{r}-\Delta \mathrm{r}$ from the center. Equation (17) is analogous to the definition of boundary layer thickness in viscous flow theory, where the significant share of viscous forces is concentrated in the boundary layer region.
However in this case, $\Delta \mathrm{r}$ has been defined as the region where $90 \%$ of the radial density variation is accumulated. With the above description of $\Delta \mathrm{r}$, the flow has been modeled as two density regimes:

$$
\begin{gathered}
\rho_{c o}(\text { core region })=\bar{\rho}_{\text {cto } r-\Delta r}, \\
\rho_{\text {an }}(\text { annular region })=\bar{\rho}_{r-\Delta r \text { to } w} .
\end{gathered}
$$

Here, Eqs. (18) and (19) show that $\rho_{c o}$ and $\rho_{a n}$ are equal to the average density $\left(\bar{\rho}_{\text {radial distance }}\right)$ in the corresponding cross section region as shown in Fig. 12. So, given the simplified flow description, the buoyant forces can be calculated for small axial element $(\Delta \mathrm{x})$. Accordingly, the net buoyant forces are defined in terms of force per unit crosssectional area (CA) using Eq. (20) with an assumption that radial density profile remains the same throughout the small axial element length $(\Delta \mathrm{x})$ considered for the analysis

$$
\Delta p_{b}=\frac{F_{b}(\text { Net Buoyancy force })}{C A} \sim\left(\rho_{c o}-\rho_{a n}\right) g \Delta x\left(\frac{C A_{\Delta r}}{C A}\right) .
$$

(2) The fluid experiences acceleration effects throughout the cross section as its genesis lies in the axial density variation. In other words, more the density gradient downstream; higher is the fluid acceleration required to maintain the same mass flow rate. As displayed in Fig. 12 (flow side view), the actual flow has been restructured to have a single density value at every axial location, which is the average cross-sectional density $(\bar{\rho})$. Hence, acceleration effects are enumerated as the difference in the inertia force per unit cross-sectional area shown in the following equation:

$$
\Delta p_{a}=\frac{F_{a}(\text { Net inertia force })}{C A} \sim\left(\bar{\rho}_{x_{2}} U_{x_{2}}^{2}-\bar{\rho}_{x_{1}} U_{x_{1}}^{2}\right)
$$

Therefore, with proper substitution, Eq. (21) takes the following form:

$$
\Delta p_{a} \sim G^{2}\left(\frac{\bar{\rho}_{x_{1}}-\bar{\rho}_{x_{2}}}{\bar{\rho}_{x_{1}} \bar{\rho}_{x_{2}}}\right)
$$
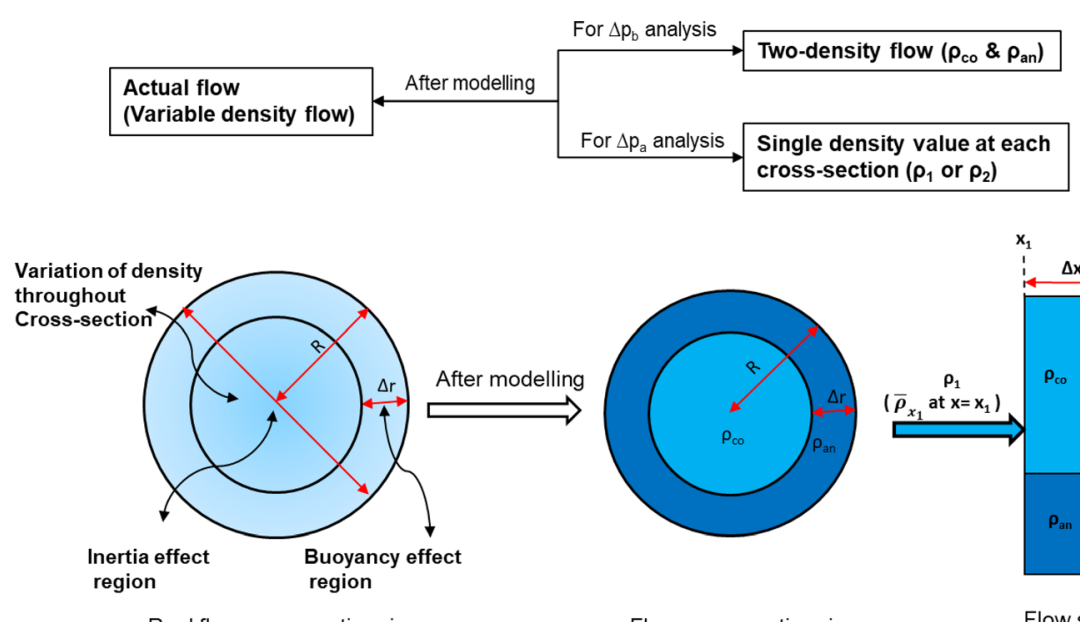

Real flow cross-section view
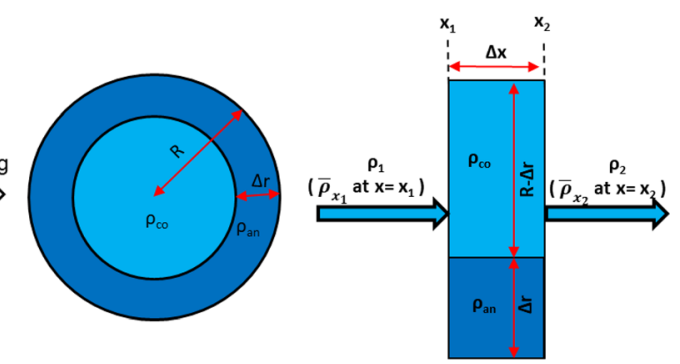

Flow cross-section view
FIG. 12. Schematic of flow simplification for the mathematical analysis. The image gives a feel of how the actual flow has been simplified for the analysis. Two types of blue color have been used to represent the modeled flow field consisting of two different regions $\left(\rho_{c o}\right.$ and $\left.\rho_{a n}\right)$ in the cross-sectional view. Besides, the side view has been included to show the modified flow used for the calculation of both the forces. 
Here, $U_{x_{1}}=$ average axial velocity at $=x_{1}, U_{x_{2}}=$ average axial velocity at $x=x_{2}$ and $\mathrm{G}$ is the mass flux.

Furthermore, the above expressions have been utilized to calculate the value of the forces at various axial locations along the flow path for all the cases. This probe has also been made more inclusive by performing the computation for case- 3 , which corresponds to Shitsman's ${ }^{5}$ experiments, which reported no HTD. Figures 13(a) and 13(b) present pressure term and the difference in the pressure terms, respectively, for all the cases based on two-phase results. It elucidates the understanding that even though both forces are present in the flow, the dominant one governs the flow.

For example, in case-2, $\Delta p_{a}$ is at least one order higher than the $\Delta p_{b}$ throughout the flow, whereas for case-1, such clarity does not exist. In other words, it can be inferred from Fig. 13(a) that at the

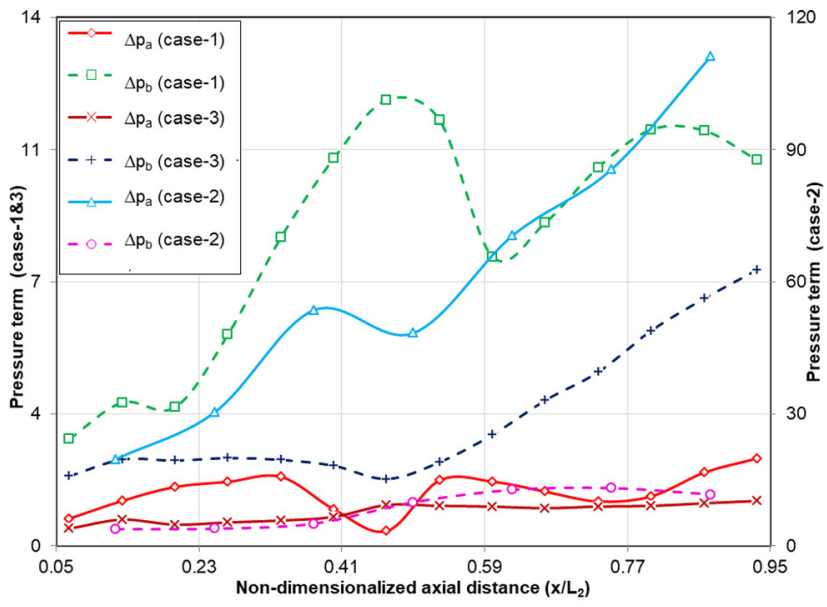

(a)

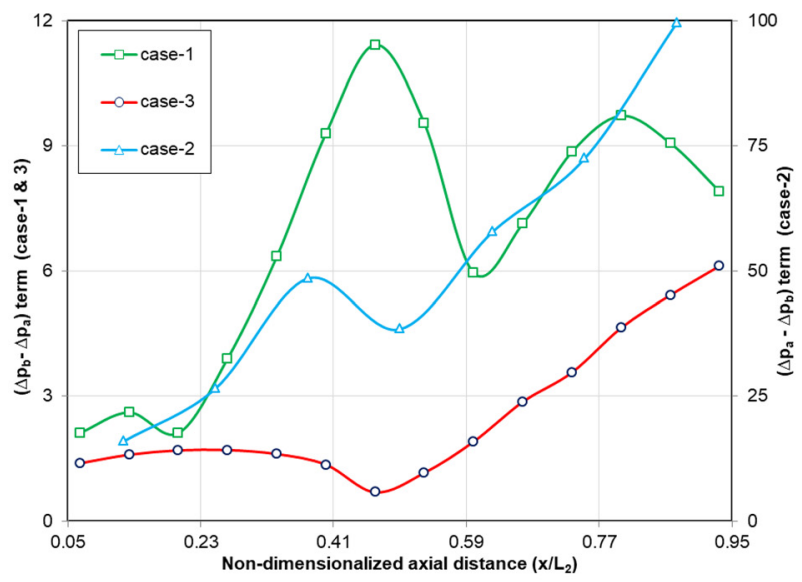

(b)

FIG. 13. Pressure terms have been mapped for different axial location based on Eqs. (20) and (22) using the two-phase results. (a) It shows pressure terms $\left(\Delta p_{b}\right.$ and $\Delta p_{a}$ ) variation for all cases. The order of magnitude is different for case-2 compared to other cases because of its high heat and mass flux. (b) It shows the difference between the two pressure components $\left(\Delta p_{b}-\Delta p_{a}\right.$ or vice versa) depending on the dominant term. location of the first HTD in case- $1, \Delta p_{b}$ exceeds the $\Delta p_{a}$ significantly but for the second HTD as discussed earlier, $\Delta p_{a}$ has a greater influence. Also, at the location of the second wall temperature peak, $\Delta p_{b}$ passes through a local minimum, whereas $\Delta p_{a}$ experiences a local maximum which is also reflected as a local minimum in the pressure difference plot, as shown in Fig. 13(b). Surprisingly, for case-3, both the forces are of the same order in the entirety of the flow domain as represented in both Figs. 13(a) and 13(b). This again leads us to a significant deduction that the interplay of both the forces dictates the supercritical flow behavior. Further, it seems that buoyancy and acceleration govern the two extremes of the supercritical flow spectrum with HTD phenomena. In contrast, when the two forces are commensurate, no HTD is reported. The explanation lies in the way the velocity profile is tempered in the presence of the two forces, as shown in Fig. 14. Since buoyancy is concentrated in the near-wall region while the bulk experiences major chunk of acceleration effect, the apparent conclusion is that the ideal behavior lies in the balance of the two factors. The heat transfer coefficient, which depends on bulk temperature $\left(T_{b}\right)$ and wall temperature $\left(T_{w}\right)$, has been plotted in Fig. 14 based on the following equations:

$$
\begin{gathered}
T_{b}(\text { Bulk temperature })=\frac{\int_{0}^{R} 2 \pi T C_{p} \rho u r d r}{\int_{0}^{R} 2 \pi C_{p} \rho u r d r}, \\
\text { heat transfer coefficient }=\frac{q}{T_{w}-T_{b}} .
\end{gathered}
$$

\section{E. Insights into volume fraction}

The most prominent advantage, the pseudophase change model, exhibits over single-fluid approach is that it explores volume fraction to unveil the heat transfer characteristics to a greater depth. It equips us with the phase distribution in the flow, which can be explored as analogous to the boiling process in subcritical fluids.

Further, it is necessary to investigate the extent of volume fraction in all the cases before reaching any conclusion. The volume fraction of the second phase (lighter fluid or VSCW) for case- 2 and case- 1 has been mapped in Figs. 15(a) and 15(b), respectively. The variations are plotted close to the wall as the volume fraction vanishes to zero within a short distance from the heated boundary. Case-2 in Fig. 15(b) shows a gradual penetration of VSCW phase in the bulk fluid, which is similar to flow boiling where liquid-vapor interface distance from the wall increase along the axial length (in the streamwise direction).

On the contrary, the volume fraction of VSCW shows a sudden jump in penetration for case- 1 at the location of the first peak. Figure 15 (a) displays this pattern at $\mathrm{x}=0.6 \mathrm{~m}$; the plot shows a steep rise in the value compared to volume fraction variation at $\mathrm{x}=0.5 \mathrm{~m}$. No such behavior is observed for the second HTD location. The jump in the LSCW-VSCW (two-phase) interface separation (Fig. 16 schematic shows phase boundary) from the heated boundary is analogous to film boiling phenomena in subcritical fluids. In other words, the moderate increase in the volume fraction of VSCW along the streamwise direction is similar to normal boiling (nucleate boiling) as in case-2. So, the transition from normal boiling to film boiling can be marked by the steep increase in the volume fraction reflected at the location of first temperature peak in case-1. Therefore, one can use the term 


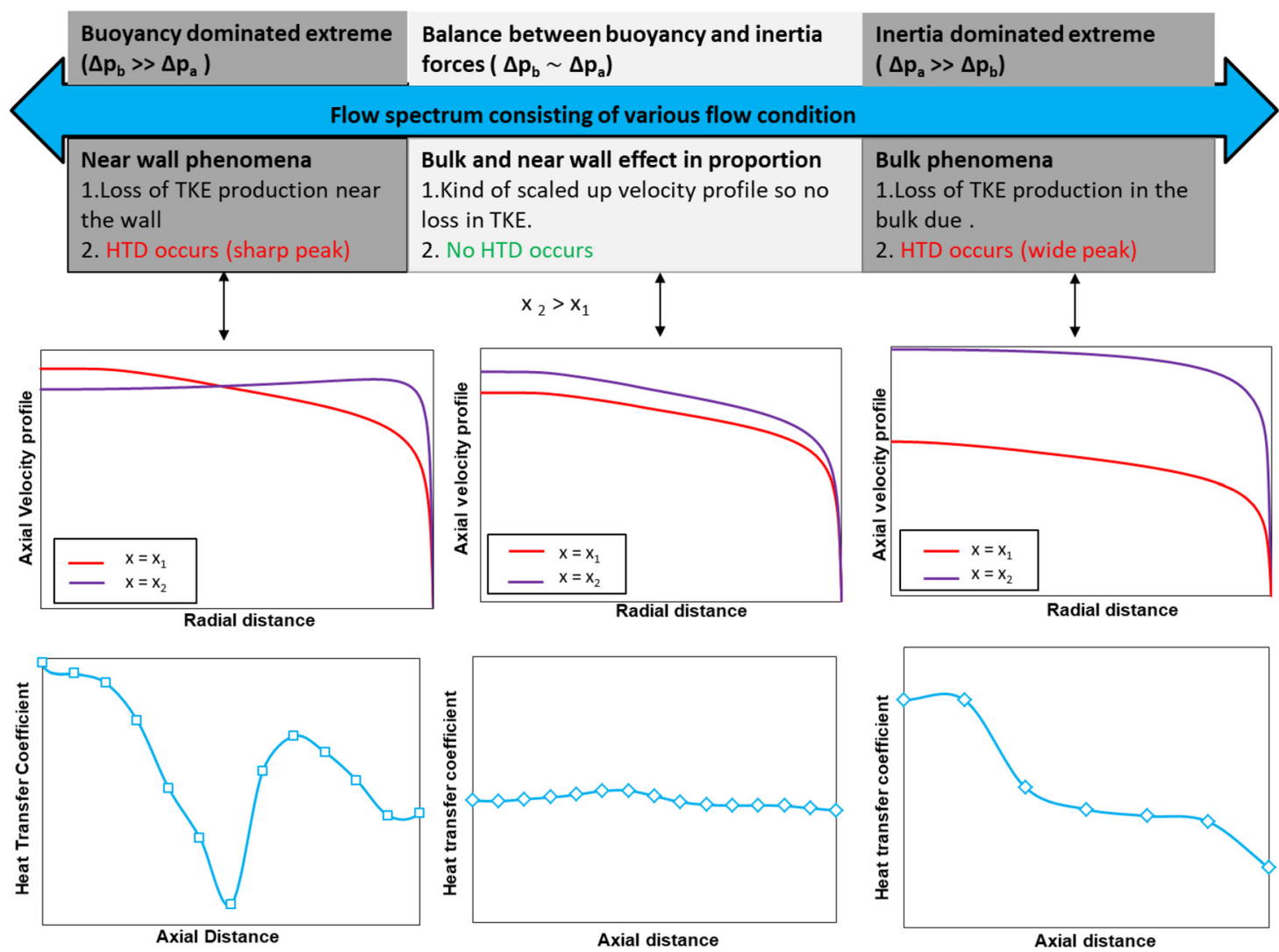

FIG. 14. Schematic depicting the various possibility of flow in supercritical fluids. The image splits the supercritical flow spectrum into three possibilities based on the dominance of the buoyancy and acceleration effects in the flow. It appears that the ideal and most desirable outcome is when the two forces are of the same order in the entire flow domain so that their cumulative effect is just to scale up the velocity without altering its gradient. Also, the heat transfer coefficient variation reveals the nature of impairment and severity of the HTD associated with the two extremes of the SCW flow spectrum.

pseudofilm boiling phenomenon for HTD caused by buoyancy solely but not for the HTD reported due to acceleration effects.

Further, a scaling analysis has been proposed for the theoretical estimation of the two-phase thickness (h) from the wall, as shown in Fig. 16. This analysis aims to accommodate all the factors which affect the pseudophase change process. It also presents $\mathrm{h}$ in a single dimensionless expression. $\mathrm{h}$ has been approached in the following way:

$$
h \propto\left\{\begin{array}{l}
\text { heat input }[q(S A)] \\
1 / \text { mass flow rate }[G(C A)] \\
1 / \text { Inlet enthalpy difference }\left(\Delta H=H_{p c}-H_{\text {in }}\right) . \\
\text { radius }(R) \\
\Delta \rho / \rho_{p c}=\left(\rho_{\text {in }}-\rho_{p c}\right) / \rho_{p c}
\end{array}\right.
$$

Here, $\rho_{i n}$ is the inlet density, $\rho_{p c}$ is the pseudocritical density, $H_{p c}$ is the pseudocritical specific enthalpy, and $H_{i n}$ is the inlet specific enthalpy.

The above equation includes $\Delta H$ since $\mathrm{T}_{\mathrm{pc}}$ has been set as the saturation temperature. Also, the last factor incorporates the dynamic nature of flow on the pseudophase change because $\Delta \rho$ term (assumed to be of the order $\rho_{\text {in }}-\rho_{\text {pc }}$ ) is common in Eqs. (20) and (22) of both the forces governing the supercritical flow. The assumed scale for the $\Delta \rho$ term is most relevant because density variation is very less beyond the pseudocritical point as the temperature rises. Equation (25) can be further simplified using Surface area $(S A)=\pi(2 R) x$, Cross-sectional area $(C A)=\pi\left(R^{2}\right)$ and grouping all the variables involved in a nondimensional form

$$
\frac{h}{R}=C\left(\frac{q x}{G R \Delta H}\right)\left(\frac{\Delta \rho}{\rho_{p c}}\right) .
$$

Here, $\mathrm{C}$ is a proportionality constant. After rearranging the above equation, it expresses a dimensionless number $(\mathrm{P})$ representing the two-phase thickness

$$
P=\left(\frac{h}{R}\right)\left(\frac{q x}{G R \Delta H}\right)\left(\frac{\Delta \rho}{\rho_{p c}}\right) .
$$

The nondimensionless number $\mathrm{P}$ has been plotted as a function of nondimensional axial length $\left(x / L_{2}\right)$ for all the cases in Fig. 17(a). The plot shows maxima for the first HTD location in case-1, whereas, for the other cases, there is a gradual increase in the value. So this peak corresponds to the jump in the volume fraction reported in case-1. 


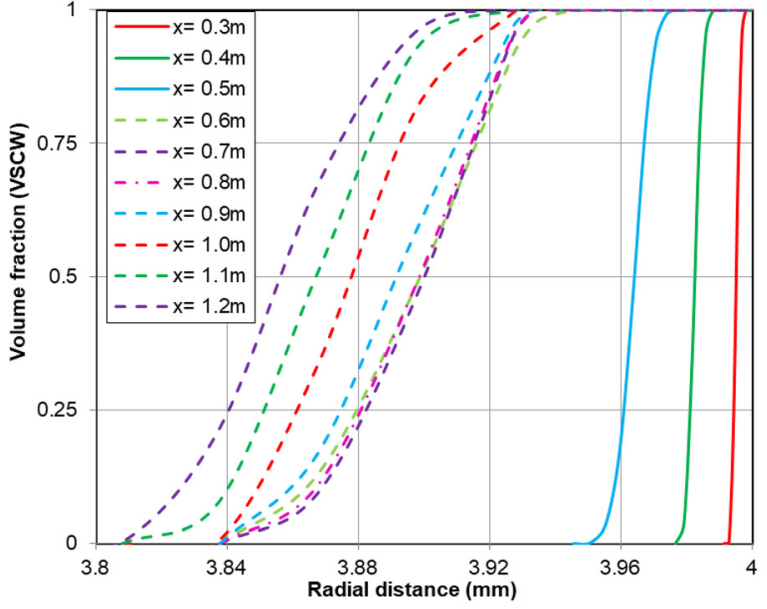

(a)

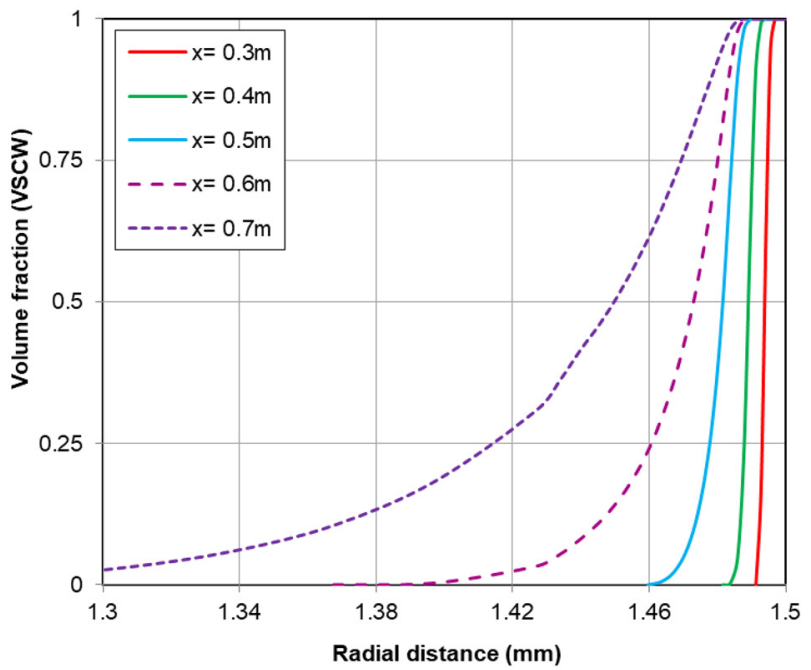

(b)

FIG. 15. Radial variation of volume fraction of VSCW (second phase) at different axial location: (a) Case-1 shows the peculiar behavior of sudden rise in VSCW volume fraction at first HTD location which is absent at the position of the second HTD. (b) Case-2 represents the monotonic increase in the VSCW volume fraction in the streamwise direction. Also, VSCW phase penetration in case-2 is greater than case-1 because of the higher heat flux and lower cross-sectional area.

Figure 17(a) further bolsters the idea of pseudofilm boiling phenomena for HTD occurrence in the buoyancy dominated flow regime.

Further, Eq. (27) is an expression that consists of all known input variables except for $\mathrm{h}$. So, the same expression can be made independent of any flow details by reframing to be capable of predicting the two-phase thickness (or the two-phase interface distance from the wall) theoretically $\left(\mathrm{h}_{\mathrm{th}}\right)$ instead of using numerical $h$ value $\left(h_{n u}\right)$.

We also offer some alternate definitions as follows:

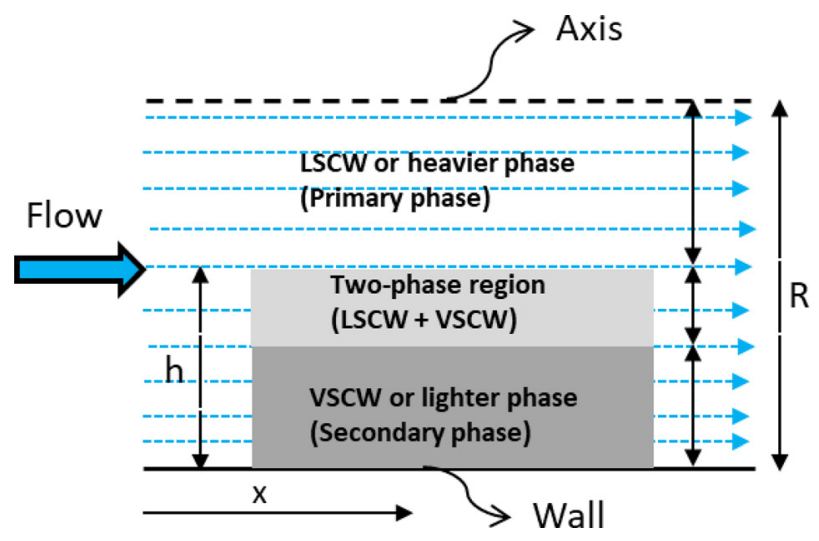

FIG. 16. Pseudo-two-phase flow representation near the wall marking different phases (LSCW and VSCW) and their boundaries in the radial direction at any axial location. Also, since the proposed approach is similar to flow boiling in the subcritical flows, there exists a saturation region where two phases coexist. So, the distance of the interface of this coexisting phases region (LSCW + VSCW) and LSCW from the wall has been named as two-phase thickness or $h$, which makes it a pertinent length scale for any supercritical flow.

$$
\text { Alternate definitions are }\left\{\begin{array}{l}
q=K_{e}\left(\frac{T_{w}-T_{p c}}{h}\right) \\
\Delta H=C_{p}\left(T_{p c}-T_{i n}\right) \\
m=\rho U
\end{array} .\right.
$$

Here, $K_{e}$ represents the effective thermal conductivity. The $\Delta H$ expression in Eq. (28) is justified because the flow is at constant pressure. Similarly, the form of $q$ in Eq. (28) is warranted because of the use of $K_{e}$ which accounts for turbulent contributions in thermal conductivity. However, the default assumption in this equation is that $\mathrm{h}$ is very small. So, incorporating the above alternate definitions into Eq. (27) and with rearrangement, the final appearance of the equation is as follows:

$$
h_{\text {th }}=C\left(\rho^{*}\right)^{0.5}\left(T^{*}\right)^{0.5}\left(P e_{R}\right)^{-0.5}(R x)^{0.5} .
$$

Here, $T^{*}=\frac{T_{w}-T_{p s}}{T_{p s}-T_{i n}}, \rho^{*}=\frac{\Delta \rho}{\rho_{p c}}$ and $P e_{R}$ is a Peclet number based on radius (R). The equation proves the dependency of $\mathrm{h}$ on $T^{*}, P e_{R}$, and $(x)^{0.5}$ which will vary for all axial location in the flow domain. In the end, values of $h_{t h}$ have been compared with $h_{n u}$, and the same has been plotted for $\mathrm{C}=0.1$ [since for scaling analysis, the constant should be $\mathrm{O}(1)$ ] as exhibited in Fig. 17(b). The juxtaposition reveals that Eq. (29) is robust enough to predict the value of $h$, and offers a relevant length scale for supercritical flow. Although Eq. (29) reasonably predicts the $\mathrm{h}$ values, as shown in Fig. 17(b), the disparity in the value increase for case- 2 in the downstream direction. As mentioned in the preceding paragraph, it can be attributed to the fact that expression of $q$ in Eq. (28) will be close to actual heat flux when h is very small, but for case-2, h increases significantly in the streamwise direction, and the same is reflected in the form of the deviation between $h_{t h}$ and $h_{n u}$. The validity of Eq. (29) is best suited for the cases where h is comparatively smaller $(h \ll R)$.

\section{CONCLUSION}

The heat transfer deterioration (HTD) in supercritical fluid is numerically tested and compared to results obtained in Shitsman and 


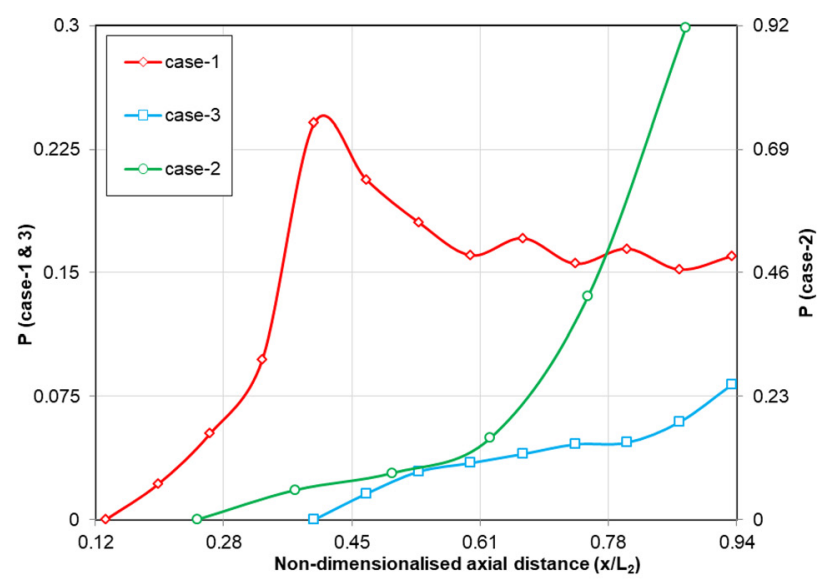

(a)

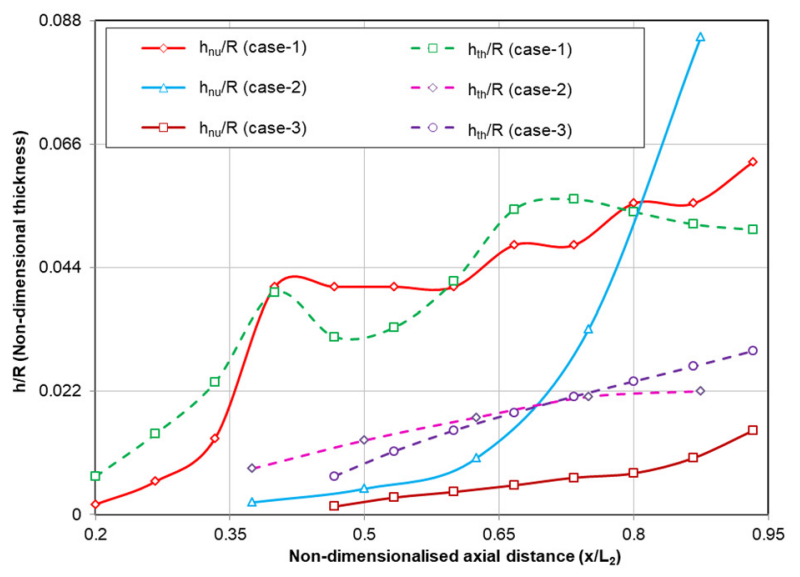

(b)

FIG. 17. It represents the value of " $h$ " in different forms as a function of nondimensionalized axial distance. (a) $\mathrm{P}$ (dimensionless two-phase thickness) shows a maximum at the position of first HTD in case-1, and for the other HTD (all the cases) shows a monotonic increase in axial direction. (b) It displays the comparison of $h$ computed from both methods (theoretical and numerical) and shows the significant match between the values of $h_{t h}$ and $h_{n u}$ for $\mathrm{C}=0.1$.

Ornatskij experiments using an unconventional pseudophase change method. This is achieved by employing the VOF multiphase model in commercial software ANSYS FLUENT. The results showed that the proposed model behaves similarly to the traditional single-fluid approach. This proposed quantitative analysis offers a more lucid and clearer picture of all the major dynamics in supercritical flows. Further, the two-phase model equips us with an opportunity to set up an analogy with the flow boiling phenomena in subcritical flows. The volume fraction variation shows a similar pattern as pseudofilm boiling for a particular kind of HTD, governed by buoyant forces. It is supported by the observed local maximum in the dimensionless twophase thickness plot. Besides, the theoretical expression for calculating dimensionless $h$ gives a better insight into the supercritical flow, and its prediction capabilities offer us an important length scale without going into intricate flow details. Although the proposed method fared well in predicting HTD, there is a scope of improvements that need to be addressed. For instance, the pseudophase change idea employs a multiphase model that has parameters that need to be fine-tuned for the supercritical fluid applications by extensively employing two-phase method for SF flow. Even a different phase-change model (other than the Lee model) along with an improved turbulence model (like using variable turbulent Prandtl number) can be used to implement the proposed two-phase method. Also, the parameter that is introduced during the pseudophase change process, such as $\Delta \mathrm{T}$, has to be explored. In addition, the two-phase approach should be implemented for various SF flow conditions reported in the literature to refine the model and judge the performance against the conventional single-phase method. The same will also help in corroborating the observations made in the quantitative analysis. It offers a way forward to conduct a lot of experiments whose results can be compared to the proposed model. The value of parameters for specific flow conditions can be also investigated further. In the end, the $h_{\text {th }}$ equation can be investigated further for the proportionality constant $\mathrm{C}$ and its variation in different fluids in supercritical conditions.

\section{ACKNOWLEDGMENTS}

The authors would like to acknowledge the financial support from the Department of Science and Technology (DST), Government of India, under the National Center for Clean Coal Research and Development (NCCCR\&D) scheme.

\section{DATA AVAILABILITY}

The data that support the findings of this study are available from the corresponding author upon reasonable request.

\section{REFERENCES}

${ }^{1}$ A. R. Imre, U. K. Deiters, T. Kraska, and I. Tiselj, “The pseudocritical regions for supercritical water,” Nucl. Eng. Des. 252, 179 (2012).

${ }^{2}$ W. B. Hall, "Heat transfer near the critical point," Adv. Heat Transfer 7, 1 (1971).

${ }^{3}$ D. T. Banuti, "Crossing the Widom-line-Supercritical pseudo-boiling," J. Supercrit. Fluids 98, 12 (2015).

${ }^{4}$ D. T. Banuti, M. Raju, and M. Ihme, "Fluids between supercritical liquids and gases-Reconciling dynamic and thermodynamic state transitions," J. Supercrit. Fluids 165, 104895 (2020).

${ }^{5}$ M. E. Shitsman, "Impairment of the heat transmission at supercritical pressures,” High Temp. 1, 237-244 (1963).

${ }^{6}$ H. S. Swenson, J. R. Carver, and C. R. Kakarala, "Heat transfer to supercritical water in smooth-bore tubes," J. Heat Transfer 87, 477 (1965).

${ }^{7}$ J. W. Ackerman, "Pseudoboiling heat transfer to supercritical pressure water in smooth and ribbed tubes," J. Heat Transfer 92, 490 (1970).

${ }^{8} \mathrm{~K}$. Yamagata and K. Nishikawa, "Forced convective heat transfer to supercritical water flowing in tubes," Int. J. Heat Mass Transfer 15, 2575 (1972).

${ }^{9} \mathrm{M}$. E. Shitsman, in Proceedings of the Institution of Mechanical Engineers Conference (1967), Vol. 182, p. 36.

${ }^{10}$ W. B. Hall and J. D. Jackson, "Laminarisation of a turbulent pipe flow by buoyancy forces," ASME Paper No. 69-HT-55 (1969).

${ }^{11} J$. D. Jackson, M. A. Cotton, and B. P. Axcell, "Studies of mixed convection in vertical tubes,” Int. J. Heat Fluid Flow 10, 2 (1989).

${ }^{12}$ J. D. Jackson, "Fluid flow and convective heat transfer to fluids at supercritical pressure,” Nucl. Eng. Des. 264, 24 (2013).

${ }^{13}$ B. S. Shiralkar and P. Griffith, "Deterioration in heat transfer to fluids at supercritical pressure and high heat fluxes," J. Heat Transfer 91, 27 (1969).

${ }^{14}$ S. Koshizuka, N. Takano, and Y. Oka, "Numerical analysis of deterioration phenomena in heat transfer to supercritical water," Int. J. Heat Mass Transfer 38, 3077 (1995). 
${ }^{15}$ M. T. Kao, M. Lee, Y. M. Ferng, and C. C. Chieng, "Heat transfer deterioration in a supercritical water channel," Nucl. Eng. Des. 240, 3321 (2010).

${ }^{16}$ J. H. Bae, J. Y. Yoo, and H. Choi, "Direct numerical simulation of turbulent supercritical flows with heat transfer," Phys. Fluids 17, 105104 (2005).

${ }^{17}$ J. Yang, Y. Oka, Y. Ishiwatari, J. Liu, and J. Yoo, "Numerical investigation of heat transfer in upward flows of supercritical water in circular tubes and tight fuel rod bundles," Nucl. Eng. Des. 237, 420 (2007).

${ }^{18} \mathrm{H}$. Cheng, J. Zhao, and M. K. Rowinski, "Study on two wall temperature peaks of supercritical fluid mixed convective heat transfer in circular tubes," Int. J. Heat Mass Transfer 113, 257 (2017).

${ }^{19}$ Q. L. Wen and H. Y. Gu, "Numerical investigation of acceleration effect on heat transfer deterioration phenomenon in supercritical water," Prog. Nucl. Energy 53, 480 (2011).

${ }^{20}$ J. H. Bae, J. Y. Yoo, and D. M. McEligot, "Direct numerical simulation of heated CO2 flows at supercritical pressure in a vertical annulus at $\operatorname{Re}=8900$," Phys. Fluids 20, 055108 (2008).

${ }^{21} \mathrm{H}$. Wang, L. K. H. Leung, W. Wang, and Q. Bi, “A review on recent heat transfer studies to supercritical pressure water in channels," Appl. Therm. Eng. 142, 573 (2018).

${ }^{22} \mathrm{M}$. Pizzarelli, "The status of the research on the heat transfer deterioration in supercritical fluids: A review," Int. Commun. Heat Mass Transfer 95, 132 (2018).

${ }^{23}$ B. Zhu, J. Xu, X. Wu, J. Xie, and M. Li, "Supercritical "boiling" number, a new parameter to distinguish two regimes of carbon dioxide heat transfer in tubes," Int. J. Therm. Sci. 136, 254 (2019).
${ }^{24}$ B. Zhu, J. Xu, C. Yan, and J. Xie, "The general supercritical heat transfer correlation for vertical up-flow tubes: K number correlation,” Int. J. Heat Mass Transfer 148, 119080 (2020).

${ }^{25}$ N. K. Singh and B. Premachandran, "Saturated film boiling over a circular cylinder subjected to horizontal cross-flow in the mixed regime," Phys. Fluids $\mathbf{3 1}$ 082109 (2019).

${ }^{26}$ N. K. Singh and B. Premachandran, "Mixed regime of film boiling over a horizontal cylinder in an upward flow of saturated liquid," Phys. Fluids 30, 122101 (2018).

${ }^{27}$ C. Keepaiboon, A. S. Dalkilic, O. Mahian, H. S. Ahn, S. Wongwises, P. K. Mondal, and M. S. Shadloo, "Two-phase flow boiling in a microfluidic channel at high mass flux," Phys. Fluids 32, 093309 (2020).

${ }^{28}$ D. Ricci, P. Natale, and F. Battista, "Experimental and numerical investigation on the behaviour of methane in supercritical conditions," Appl. Therm. Eng 107, 1334 (2016).

${ }^{29} \mathrm{~S}$. W. J. Welch and J. Wilson, "A volume of fluid based method for fluid flows with phase change," J. Comput. Phys. 160, 662 (2000).

${ }^{30}$ Z. Pan, J. A. Weibel, and S. V. Garimella, "A saturated-interface-volume phase change model for simulating flow boiling," Int. J. Heat Mass Transfer 93, 945 (2016).

${ }^{31}$ M. Magnini and J. R. Thome, "Computational study of saturated flow boiling within a microchannel in the slug flow regime," J. Heat Transfer 138, 1 (2016).

${ }^{32}$ W. H. Lee, in Computational Methods for Two-Phase Flow and Particle Transport (World Scientific, 2013), pp. 61-82. 